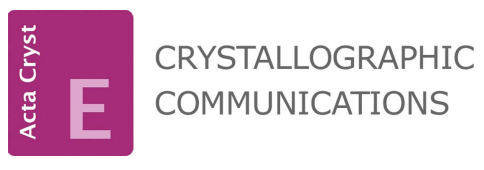

ISSN 2056-9890

Received 22 March 2019

Accepted 28 April 2019

Edited by A. V. Yatsenko, Moscow State University, Russia

Keywords: crystal structure; arylhydrazone; $\mathrm{Cu}^{\text {II }}$ chelate; two-component crystal; molecular layer formation; $\mathrm{C}-\mathrm{H} \cdots \mathrm{O}$ hydrogen bonding.

CCDC reference: 1912679

Supporting information: this article has supporting information at journals.iucr.org/e

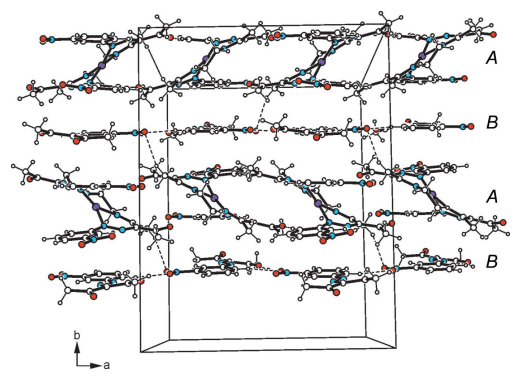

OPEN $\odot$ ACCESS

\section{Crystal structure of $\left(N, N^{\prime}\right.$-ethylenebis\{3-[2-(3- nitrophenyl)hydrazin-1-ylidene]-4-oxopentan-2- iminato])copper(II)-3-[2-(3-nitrophenyl)hydrazin- 1-ylidene]pentane-2,4-dione (1/1)}

\author{
Jan Marten, Wilhelm Seichter and Edwin Weber*
}

TU Bergakademie Freiberg, Leipziger Str. 29, D-09596 Freiberg/Sachsen, Germany. *Correspondence e-mail: edwin.weber@chemie.tu-freiberg.de

In the title 1:1 co-crystal, $\left[\mathrm{Cu}\left(\mathrm{C}_{24} \mathrm{H}_{24} \mathrm{~N}_{8} \mathrm{O}_{6}\right)\right] \cdot \mathrm{C}_{11} \mathrm{H}_{11} \mathrm{~N}_{3} \mathrm{O}_{4}$, each of the crystal components forms undulating layers which stack alternately along the $b$-axis direction. Molecules of the $\mathrm{Cu}^{\mathrm{II}}$ complex are connected via $\mathrm{C}-\mathrm{H} \cdots \mathrm{O}$ hydrogen bonds involving the nitro and keto oxygen atoms, thus forming supramolecular networks. Molecules of the arylhydrazone component are linked by $\mathrm{C}-\mathrm{H} \cdots \mathrm{O}$ interactions into zigzag strands showing no interstrand association.

\section{Chemical context}

Hydrazone imines derived from $\beta$-diketones and aryldiazonium salts using a Japp-Klingemann route (Phillips, 1959) have attracted considerable interest as precursors of potential antidiabetic drugs (Garg \& Prakash, 1971; Küçükgüzel et al., 1999) as well as regarding their particular property of hydrogen bonding (Marten et al., 2007; Sethukumar \& Arul Prakasam, 2010) and their remarkable behavior in the formation of metal complexes. Transition-metal chelates of the respective hydrazine imines have been described in great numbers (Albert et al., 1997; Mishra et al., 2000; Marten et al., 2005). Preferentially, the chelates with $\mathrm{Cu}^{\mathrm{II}}, \mathrm{Co}^{\mathrm{II}}$ and $\mathrm{Ni}^{\mathrm{II}}$ show a tetrahedrally distorted square $\mathrm{N}_{2} \mathrm{O}_{2}$ coordination environment. In the presence of a diamine and $\mathrm{Ni}^{\mathrm{II}}$, related bis(hydrazonoimine) complexes are formed displaying an unusual behavior of the effective magnetic moment at low temperature (Khudina et al., 2007). A corresponding chelate complex, formed of 3-[2-(3-nitrophenyl)hydrazin-1-ylidene]pentane-2,4-dione (Marten et al., 2018) and bis(ethylenediamine)copper(II) chloride yielded a co-crystal consisting of $N, N^{\prime}$-ethylenebis\{3-[2-(3-nitrophenyl)hydrazine-1-ylidene]-4oxopentane-2-iminato\}copper(II) and 3-[2-(3-nitrophenyl)hydrazine-1-ylidene]pentane-2,4-dione in a 1:1 stoichiometric composition (the title compound), whose crystal structure is reported on herein.

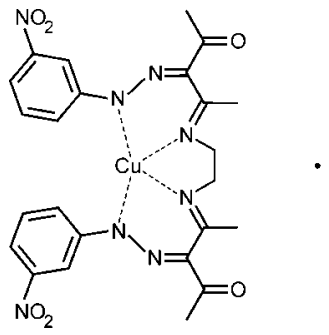



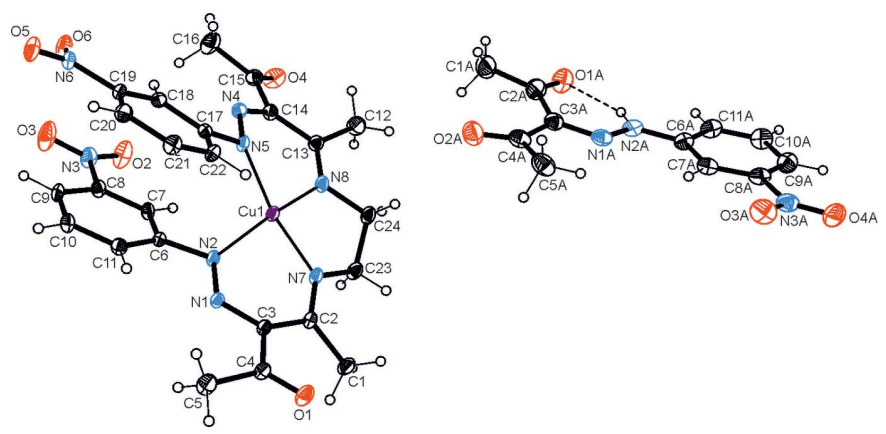

Figure 1

Perspective view of an asymmetric unit of the title co-crystal with atom labeling. Displacement ellipsoids of non-H atoms are shown at the $40 \%$ probability level.

\section{Structural commentary}

The title co-crystal possesses orthorhombic symmetry (space group $P b c a$ ) with one molecule of the $\mathrm{Cu}^{\mathrm{II}}$ complex and one molecule of the arylhydrazone in the asymmetric unit. A perspective view is shown in Fig. 1. The metal center of the complex adopts a tetrahedrally distorted square coordination environment formed by four nitrogen atoms (N2, N4, N7, N8) of the ligand. As a result of the tetradentate coordination mode and the steric interaction between the terminal aromatic rings of the ligand, the complex molecule adopts a helical geometry with a distance of 3.384 (4) A between the benzene ring centroids and a dihedral angle of $10.43(4)^{\circ}$ between the benzene ring planes. The $\mathrm{Cu}-\mathrm{N}$ distances are $1.940(2)$, 1.943 (2), 1.953 (2) and 1.957 (2) $\AA$, the bond angles N7$\mathrm{Cu}-\mathrm{N} 8, \mathrm{~N} 7-\mathrm{Cu}-\mathrm{N} 2, \mathrm{~N} 8-\mathrm{Cu}-\mathrm{N} 5$ and $\mathrm{N} 2-\mathrm{Cu}-\mathrm{N} 5$ are $86.3(1), 88.4(1), 89.1(1)$ and $100.4(1)^{\circ}$, respectively. The nitro groups deviate slightly from the planes of the respective benzene rings, with plane $\mathrm{N} 3 / \mathrm{O} 2 / \mathrm{O} 3$ being inclined to benzene ring $\mathrm{C} 6-\mathrm{C} 11$ of $3.8(1)^{\circ}$ and plane $\mathrm{N} 6 / \mathrm{O} 5 / \mathrm{O} 6$ being inclined to benzene ring $\mathrm{C} 17-\mathrm{C} 22$ by $5.1(2)^{\circ}$ between the nitro groups and the respective benzene rings. The conformation of the

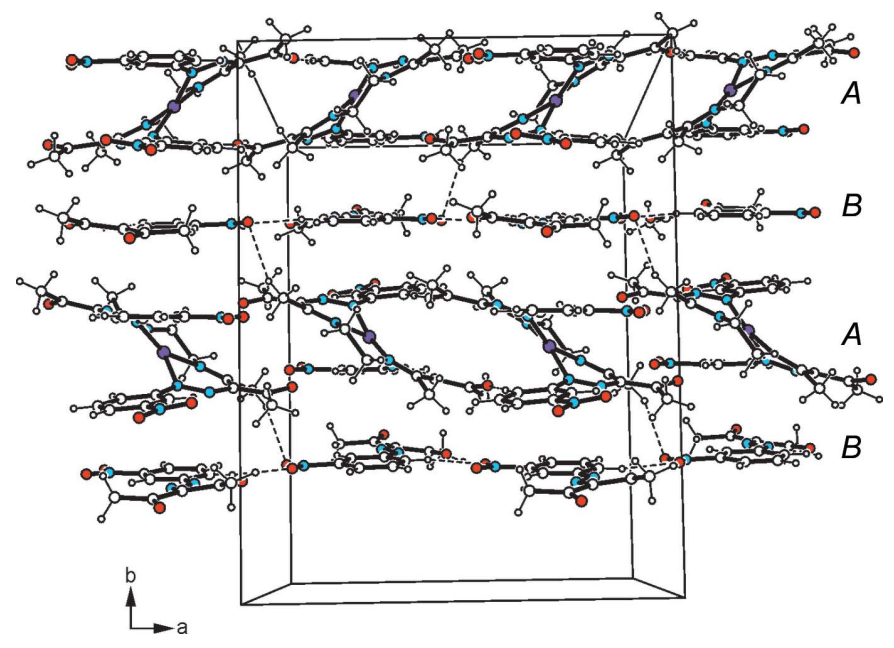

Figure 2

Packing structure of the of the title co-crystal viewed down the crystallographic $c$ axis. Dashed lines represent hydrogen bonds.
Table 1

Hydrogen-bond geometry $\left(\AA,^{\circ}\right)$.

\begin{tabular}{lllll}
\hline$D-\mathrm{H} \cdots A$ & $D-\mathrm{H}$ & $\mathrm{H} \cdots A$ & $D \cdots A$ & $D-\mathrm{H} \cdots A$ \\
\hline $\mathrm{C} 11 A-\mathrm{H} 11 A \cdots \mathrm{O} 4 A^{\mathrm{i}}$ & 0.95 & 2.43 & $3.320(3)$ & 156 \\
$\mathrm{~N} 2 A-\mathrm{H} 2 A \cdots \mathrm{O} 1 A$ & $0.90(1)$ & $1.81(2)$ & $2.531(3)$ & $135(3)$ \\
$\mathrm{C} 22-\mathrm{H} 22 \cdots \mathrm{O} 1^{\mathrm{ii}}$ & 0.95 & 2.27 & $3.207(3)$ & 169 \\
$\mathrm{C} 16-\mathrm{H} 16 A \cdots \mathrm{O} 5^{\mathrm{iii}}$ & 0.98 & 2.63 & $3.532(3)$ & 154 \\
$\mathrm{C} 12-\mathrm{H} 12 B \cdots{ }^{\text {iv }}$ & 0.98 & 2.53 & $3.413(3)$ & 151 \\
$\mathrm{C} 11-\mathrm{H} 11 \cdots 3^{\mathrm{v}}$ & 0.95 & 2.50 & $3.350(3)$ & 150 \\
$\mathrm{C} 5-\mathrm{H} 5 C \cdots \mathrm{O} 3^{\mathrm{v}}$ & 0.98 & 2.65 & $3.426(3)$ & 137 \\
$\mathrm{C} 1-\mathrm{H} 1 B \cdots \mathrm{O} 3 A^{\mathrm{vi}}$ & 0.98 & 2.58 & $3.490(3)$ & 154 \\
$\mathrm{C} 1-\mathrm{H} 1 B \cdots \mathrm{O} 1$ & 0.98 & 2.34 & $2.842(3)$ & 111 \\
\hline
\end{tabular}

Symmetry codes: (i) $x+\frac{1}{2},-y+\frac{1}{2},-z+2$; (ii) $-x,-y,-z+1$; (iii) $x+\frac{1}{2}, y,-z+\frac{1}{2}$; (iv) $x+\frac{1}{2}, y,-z+\frac{3}{2} ;$ (v) $x-\frac{1}{2}, y,-z+\frac{1}{2}$; (vi) $-x, y-\frac{1}{2},-z+\frac{3}{2}$.

arylhydrazone component is nearly identical with that found in the reported structure of this compound (Marten et al., 2018). The molecule features an intramolecular $\mathrm{N}-$ $\mathrm{H} \cdots \mathrm{O}=\mathrm{C}$ interaction that yields a six-membered hydrogenbonded ring. The dihedral angle between the mean plane of this ring and the aromatic ring is $7.8(2)^{\circ}$. The nitro group is tilted at an angle of $5.8(2)^{\circ}$ with respect to the benzene ring.

\section{Supramolecular features}

In the crystal, the $\mathrm{Cu}^{\mathrm{II}}$ complexes as well as the arylhydrazone molecules form undulating layers extending parallel to the $a c$ plane and arranged in an alternating order along the $b$-axis direction (Fig. 2). Within a layer of complexes, one carbonyl oxygen and one nitro group per molecule interact via $\mathrm{C}_{\text {arene }}-$ H..O hydrogen bonding (Desiraju \& Steiner, 1999), thus generating a supramolecular network (Table 1, Fig. 3). The arylhydrazone molecules are connected by means of $\mathrm{C}_{\text {aryl }}-$ $\mathrm{H} \cdots \mathrm{O}_{\text {nitro }}$ interactions to form zigzag-like strands that run along the $a$-axis direction (Table 1, Fig. 4). No directed noncovalent bonds are observed between the supramolecular strands. In the stacking direction, the molecules are linked by $\mathrm{C}-\mathrm{H} \cdots \mathrm{O}$ interactions involving a nitro oxygen atom of the

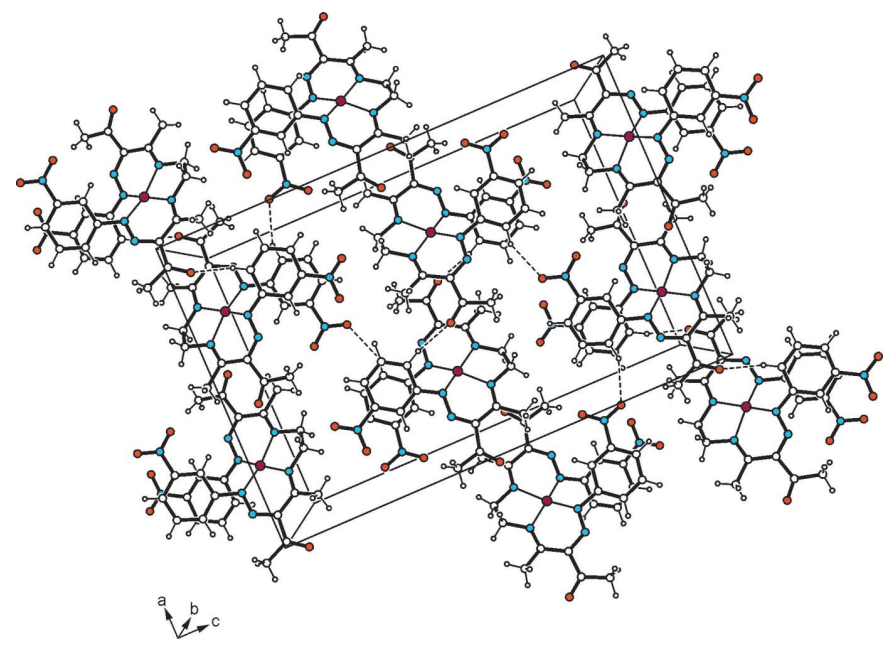

Figure 3

Structure of the $\mathrm{Cu}^{\mathrm{II}}$ complex layer viewed along the $b$ axis. The $\mathrm{C}-$ $\mathrm{H} \cdots \mathrm{O}$ interactions (Table 1) are shown as dashed lines. 


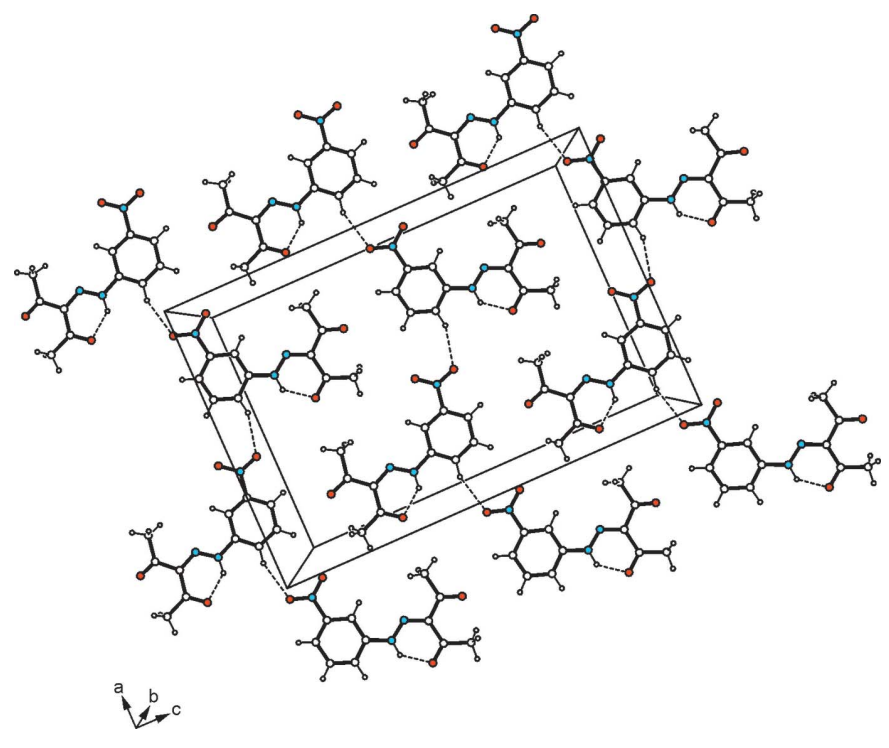

Figure 4

Layer structure of the arylhydrazone viewed along the $b$ axis. Dashed lines represent the $\mathrm{C}-\mathrm{H} \cdots \mathrm{O}$ interactions (Table 1).

arylhydrazone molecule and a methyl hydrogen of the coordinated ligand.

\section{Database survey}

A search in the Cambridge Structural Database (CSD, Version 5.38, update February 2017; Groom et al., 2016) revealed one hit for a crystal structure of a transition-metal complex containing a structurally related ligand species. The complex $N, N^{\prime}$-ethylene-bis[3-(4-methylphenyl)hydrazono-4-oxo-5,5,6,6,7,7,8,8-octafluorooctane-2-iminato]nickel(II) (JIXQAJ; Khudina et al., 2007) adopts a helical geometry that resembles that of the title complex. As a result of the presence of two extended fluoroalkyl moieties, the pattern of intermolecular non-covalent bonding is dominated by $\mathrm{C}-\mathrm{H} \cdots \mathrm{F}$ and $\mathrm{F} \cdots \mathrm{F}$ interactions (Reichenbächer et al., 2005), creating a threedimensional supramolecular architecture. Unlike the title structure, in the reported crystal structure of 3-[2-(3-nitrophenyl)hydrazine-1-ylidene]pentane-2,4-dione (Marten et al., 2018) the molecules are connected via $\mathrm{C}_{\text {arene }}-\mathrm{H} \cdots \mathrm{O}_{\text {nitro }}$ and $\mathrm{C}_{\text {arene }}-\mathrm{H} \cdots \mathrm{O}_{\text {keto }}$ interactions giving rise to supramolecular sheets.

\section{Synthesis and crystallization}

A solution containing 3-[2-(3-nitrophenyl)hydrazine-1-ylidene]pentane-2,4-dione and bis(ethylenediamine)copper(II) chloride in $n$-butanol was heated for several hours. After cooling and storing the reaction solution, blue-colored crystals could be isolated which turned out to consist of the title compound.
Table 2

Experimental details.

\begin{tabular}{|c|c|}
\hline \multicolumn{2}{|l|}{ Crystal data } \\
\hline Chemical formula & {$\left[\mathrm{Cu}\left(\mathrm{C}_{24} \mathrm{H}_{24} \mathrm{~N}_{8} \mathrm{O}_{6}\right)\right] \cdot \mathrm{C}_{11} \mathrm{H}_{11} \mathrm{~N}_{3} \mathrm{O}_{4}$} \\
\hline$M_{\mathrm{r}}$ & 833.28 \\
\hline Crystal system, space group & Orthorhombic, $\mathrm{Pbca}$ \\
\hline Temperature $(\mathrm{K})$ & 153 \\
\hline$a, b, c(\AA)$ & $\begin{array}{l}15.5820(5), 20.0517(7), \\
23.3082(8)\end{array}$ \\
\hline$V\left(\AA^{3}\right)$ & $7282.5(4)$ \\
\hline$Z$ & 8 \\
\hline Radiation type & Мo $K \alpha$ \\
\hline$\mu\left(\mathrm{mm}^{-1}\right)$ & 0.68 \\
\hline Crystal size $(\mathrm{mm})$ & $0.54 \times 0.44 \times 0.07$ \\
\hline \multicolumn{2}{|l|}{ Data collection } \\
\hline Diffractometer & Bruker SMART APEXII \\
\hline Absorption correction & $\begin{array}{l}\text { Multi-scan (SADABS; Bruker, } \\
\text { 2008) }\end{array}$ \\
\hline$T_{\min }, T_{\max }$ & $0.712,0.954$ \\
\hline $\begin{array}{l}\text { No. of measured, independent and } \\
\text { observed }[I>2 \sigma(I)] \text { reflections }\end{array}$ & $86372,9862,6112$ \\
\hline$R_{\text {int }}$ & 0.106 \\
\hline$(\sin \theta / \lambda)_{\max }\left(\AA^{-1}\right)$ & 0.687 \\
\hline \multicolumn{2}{|l|}{ Refinement } \\
\hline$R\left[F^{2}>2 \sigma\left(F^{2}\right)\right], w R\left(F^{2}\right), S$ & $0.047,0.130,0.96$ \\
\hline No. of reflections & 9862 \\
\hline No. of parameters & 524 \\
\hline No. of restraints & 1 \\
\hline $\mathrm{H}$-atom treatment & $\begin{array}{l}\mathrm{H} \text { atoms treated by a mixture of } \\
\text { independent and constrained }\end{array}$ \\
\hline$\Delta \rho_{\max }, \Delta \rho_{\min }\left(\mathrm{e} \AA^{-3}\right)$ & $1.01,-0.68$ \\
\hline
\end{tabular}

Computer programs: APEX2 and SAINT (Bruker, 2014), SHELXS97 and SHELXTL (Sheldrick, 2008), SHELXL2014 (Sheldrick, 2015) and ORTEP-3 for Windows (Farrugia, 2012).

\section{Refinement}

Crystal data, data collection and structure refinement details are summarized in Table 2. The NH H atom of 3-[2-(3-nitrophenyl)-hydrazine-1-ylidene]pentane-2,4-dione was located in a difference-Fourier map and freely refined. The C-bound and $\mathrm{N}$-bound $\mathrm{H}$ atoms were included in the model in calculated positions and refined as riding atoms: $\mathrm{C}-\mathrm{H}=0.95-0.99 \AA$ with $U_{\text {iso }}(\mathrm{H})=1.5 U_{\text {eq }}(\mathrm{C})$ for methyl and $U_{\text {iso }}(\mathrm{H})=1.2 U_{\text {eq }}(\mathrm{C})$ for other $\mathrm{H}$ atoms.

\section{References}

Albert, J., González, A., Granell, J., Moragas, R., Puerta, C. \& Valerga, P. (1997). Organometallics, 16, 3775-3778.

Bruker (2008). SADABS. Bruker AXS Inc., Madison, Wisconsin, USA.

Bruker (2014). APEX2 and SAINT. Bruker AXS Inc., Madison, Wisconsin, USA.

Desiraju, G. R. \& Steiner, T. (1999). The Weak Hydrogen Bond. Oxford University Press.

Farrugia, L. J. (2012). J. Appl. Cryst. 45, 849-854.

Garg, H. G. \& Prakash, C. (1971). J. Pharm. Sci. 60, 323-325.

Groom, C. R., Bruno, I. J., Lightfoot, M. P. \& Ward, S. C. (2016). Acta Cryst. B72, 171-179.

Khudina, O. G., Shchegol'kov, E. V., Burgart, V., Saloutin, V. I., Bukhvalov, D. V., Starichenko, D. V., Shvachko, Yu. N., Korolev, A. V., Ustinov, V. V., Aleksandrov, G. G., Eremenko, I. L., Kazheva, O. N., Shilov, G. V., D’yachenko, O. A. \& Chupakhin, O. N. (2007). Russ. Chem. Bull. 56, 108-114. 
Küçükgüzel, G., Rollas, S., Küçükgüzel, I. \& Kiraz, M. (1999). Eur. J. Med. Chem. 34, 1093-1100.

Marten, J., Seichter, W. \& Weber, E. (2005). Z. Anorg. Allg. Chem. 631, 869-877.

Marten, J., Seichter, W. \& Weber, E. (2018). IUCrData, 3, x181344.

Marten, J., Seichter, W., Weber, E. \& Böhme, U. (2007). J. Phys. Org. Chem. 20, 716-731.

Mishra, L., Yadaw, A. K., Srivastava, S. \& Bahadur Patel, A. (2000). New J. Chem. 24, 505-510.
Phillips, R. R. (1959). Org. React. 10, 143-178.

Reichenbächer, K., Süss, H. I. \& Hulliger, J. (2005). Chem. Soc. Rev. 34, 22-30.

Sethukumar, A. \& Arul Prakasam, B. (2010). J. Mol. Struct. 963, 250257.

Sheldrick, G. M. (2008). Acta Cryst. A64, 112-122.

Sheldrick, G. M. (2015). Acta Cryst. A71, 3-8. 


\section{supporting information}

Acta Cryst. (2019). E75, 834-837 [https://doi.org/10.1107/S2056989019005838]

Crystal structure of ( $N, N^{\prime}$-ethylenebis\{3-[2-(3-nitrophenyl)hydrazin-1-ylidene]-4-oxopentan-2-iminato\})copper(II)-3-[2-(3-nitrophenyl)hydrazin-1-ylidene]pentane-2,4-dione (1/1)

\section{Jan Marten, Wilhelm Seichter and Edwin Weber}

\section{Computing details}

Data collection: APEX2 (Bruker, 2014); cell refinement: SAINT (Bruker, 2014); data reduction: SAINT (Bruker, 2014); program(s) used to solve structure: SHELXS97 (Sheldrick, 2008); program(s) used to refine structure: SHELXL2014 (Sheldrick, 2015); molecular graphics: ORTEP-3 for Windows (Farrugia, 2012); software used to prepare material for publication: SHELXTL (Sheldrick, 2008).

( $N, N^{\prime}$-Ethylenebis\{3-[2-(3-nitrophenyl) hydrazin-1-ylidene]-4-oxopentan-2-iminato\})copper(II)-3-[2-(3nitrophenyl)hydrazine-1-ylidene]pentane-2,4-dione (1/1)

\section{Crystal data}

$\left[\mathrm{Cu}\left(\mathrm{C}_{24} \mathrm{H}_{24} \mathrm{~N}_{8} \mathrm{O}_{6}\right)\right] \cdot \mathrm{C}_{11} \mathrm{H}_{11} \mathrm{~N}_{3} \mathrm{O}_{4}$

$M_{r}=833.28$

Orthorhombic, $\mathrm{Pbca}$

$a=15.5820(5) \AA$

$b=20.0517$ (7) $\AA$

$c=23.3082(8) \AA$

$V=7282.5(4) \AA^{3}$

$Z=8$

$F(000)=3448$

Data collection

Bruker SMART APEXII

diffractometer

$\varphi$ and $\omega$ scans

Absorption correction: multi-scan

(SADABS; Bruker, 2008)

$T_{\min }=0.712, T_{\max }=0.954$

86372 measured reflections

Refinement

Refinement on $F^{2}$

Least-squares matrix: full

$R\left[F^{2}>2 \sigma\left(F^{2}\right)\right]=0.047$

$w R\left(F^{2}\right)=0.130$

$S=0.96$

9862 reflections

524 parameters

1 restraint
$D_{\mathrm{x}}=1.520 \mathrm{Mg} \mathrm{m}^{-3}$

Mo $K \alpha$ radiation, $\lambda=0.71073 \AA$

Cell parameters from 8051 reflections

$\theta=2.2-28.2^{\circ}$

$\mu=0.68 \mathrm{~mm}^{-1}$

$T=153 \mathrm{~K}$

Plate, blue

$0.54 \times 0.44 \times 0.07 \mathrm{~mm}$

9862 independent reflections

6112 reflections with $I>2 \sigma(I)$

$R_{\text {int }}=0.106$

$\theta_{\text {max }}=29.3^{\circ}, \theta_{\min }=2.2^{\circ}$

$h=-20 \rightarrow 21$

$k=-27 \rightarrow 25$

$l=-31 \rightarrow 28$

Primary atom site location: structure-invariant direct methods

Secondary atom site location: difference Fourier map

Hydrogen site location: mixed

$\mathrm{H}$ atoms treated by a mixture of independent and constrained refinement 
$w=1 /\left[\sigma^{2}\left(F_{\mathrm{o}}^{2}\right)+(0.0736 P)^{2}+0.2354 P\right]$

where $P=\left(F_{\mathrm{o}}^{2}+2 F_{\mathrm{c}}{ }^{2}\right) / 3$

$(\Delta / \sigma)_{\max }=0.001$

$$
\Delta \rho_{\max }=1.00 \mathrm{e} \AA^{-3}
$$$$
\Delta \rho_{\min }=-0.67 \text { e } \AA^{-3}
$$

\section{Special details}

Geometry. All esds (except the esd in the dihedral angle between two 1.s. planes) are estimated using the full covariance matrix. The cell esds are taken into account individually in the estimation of esds in distances, angles and torsion angles; correlations between esds in cell parameters are only used when they are defined by crystal symmetry. An approximate (isotropic) treatment of cell esds is used for estimating esds involving 1.s. planes.

Fractional atomic coordinates and isotropic or equivalent isotropic displacement parameters $\left(\AA^{2}\right)$

\begin{tabular}{|c|c|c|c|c|}
\hline & $x$ & $y$ & $z$ & $U_{\text {iso }} * / U_{\text {eq }}$ \\
\hline $\mathrm{Cu} 1$ & $0.23540(2)$ & $0.01555(2)$ & $0.46535(2)$ & $0.01874(9)$ \\
\hline $\mathrm{O} 1$ & $-0.07371(10)$ & $-0.09037(9)$ & $0.50519(7)$ & $0.0288(4)$ \\
\hline $\mathrm{O} 2$ & $0.46419(12)$ & $-0.03799(13)$ & $0.31472(9)$ & $0.0542(6)$ \\
\hline $\mathrm{O} 3$ & $0.43989(13)$ & $-0.02538(13)$ & $0.22515(9)$ & $0.0543(6)$ \\
\hline $\mathrm{O} 4$ & $0.56630(10)$ & $0.08866(10)$ & $0.47203(8)$ & $0.0357(4)$ \\
\hline O5 & $0.23463(12)$ & $0.16123(9)$ & $0.16939(7)$ & $0.0348(4)$ \\
\hline O6 & $0.35277(12)$ & $0.13307(11)$ & $0.21053(8)$ & $0.0419(5)$ \\
\hline N1 & $0.10026(11)$ & $-0.06399(9)$ & $0.41500(7)$ & $0.0171(4)$ \\
\hline N2 & $0.17823(11)$ & $-0.04081(9)$ & $0.40872(7)$ & $0.0181(4)$ \\
\hline N3 & $0.41581(13)$ & $-0.03341(11)$ & $0.27448(9)$ & $0.0293(5)$ \\
\hline N4 & $0.36211(11)$ & $0.10044(9)$ & $0.41302(8)$ & $0.0197(4)$ \\
\hline N5 & $0.27979(11)$ & $0.08627(9)$ & $0.41566(7)$ & $0.0180(4)$ \\
\hline N6 & $0.27544(13)$ & $0.14372(10)$ & $0.21161(8)$ & $0.0244(4)$ \\
\hline N7 & $0.18147(11)$ & $-0.03715(10)$ & $0.52509(7)$ & $0.0200(4)$ \\
\hline N8 & $0.31978(12)$ & $0.04022(10)$ & $0.52296(8)$ & $0.0208(4)$ \\
\hline $\mathrm{C} 1$ & $0.07728(15)$ & $-0.11055(13)$ & $0.57134(10)$ & $0.0282(5)$ \\
\hline $\mathrm{H} 1 \mathrm{~A}$ & 0.1254 & -0.1339 & 0.5895 & $0.042 *$ \\
\hline $\mathrm{H} 1 \mathrm{~B}$ & 0.0352 & -0.1432 & 0.5576 & $0.042 *$ \\
\hline $\mathrm{H} 1 \mathrm{C}$ & 0.0500 & -0.0809 & 0.5994 & $0.042 *$ \\
\hline $\mathrm{C} 2$ & $0.10993(14)$ & $-0.06983(11)$ & $0.52124(9)$ & $0.0188(4)$ \\
\hline $\mathrm{C} 3$ & $0.06458(13)$ & $-0.07253(11)$ & $0.46652(9)$ & $0.0176(4)$ \\
\hline $\mathrm{C} 4$ & $-0.02728(13)$ & $-0.09027(11)$ & $0.46301(10)$ & $0.0203(5)$ \\
\hline C5 & $-0.06491(15)$ & $-0.10480(14)$ & $0.40497(10)$ & $0.0315(6)$ \\
\hline H5A & -0.1214 & -0.1256 & 0.4096 & $0.047 *$ \\
\hline H5B & -0.0269 & -0.1352 & 0.3840 & $0.047 *$ \\
\hline $\mathrm{H} 5 \mathrm{C}$ & -0.0710 & -0.0631 & 0.3834 & $0.047 *$ \\
\hline C6 & $0.20627(13)$ & $-0.04200(11)$ & $0.35057(9)$ & $0.0175(4)$ \\
\hline $\mathrm{C} 7$ & $0.29434(13)$ & $-0.04252(11)$ & $0.34078(9)$ & $0.0191(4)$ \\
\hline $\mathrm{H} 7$ & 0.3338 & -0.0463 & 0.3717 & $0.023^{*}$ \\
\hline $\mathrm{C} 8$ & $0.32268(14)$ & $-0.03731(12)$ & $0.28491(10)$ & $0.0214(5)$ \\
\hline C9 & $0.26778(15)$ & $-0.03286(11)$ & $0.23830(10)$ & $0.0224(5)$ \\
\hline H9 & 0.2895 & -0.0283 & 0.2004 & $0.027 *$ \\
\hline $\mathrm{C} 10$ & $0.18059(15)$ & $-0.03522(11)$ & $0.24867(10)$ & $0.0227(5)$ \\
\hline H10 & 0.1415 & -0.0339 & 0.2174 & $0.027 *$ \\
\hline $\mathrm{C} 11$ & $0.14957(14)$ & $-0.03950(11)$ & $0.30421(9)$ & $0.0204(5)$ \\
\hline H11 & 0.0894 & -0.0407 & 0.3108 & $0.025^{*}$ \\
\hline
\end{tabular}




\begin{tabular}{|c|c|c|c|c|}
\hline $\mathrm{C} 12$ & $0.44975(16)$ & $0.08755(14)$ & $0.56354(11)$ & $0.0351(6)$ \\
\hline $\mathrm{H} 12 \mathrm{~A}$ & 0.4146 & 0.1030 & 0.5958 & $0.053 *$ \\
\hline H12B & 0.4891 & 0.1232 & 0.5518 & $0.053^{*}$ \\
\hline $\mathrm{H} 12 \mathrm{C}$ & 0.4829 & 0.0483 & 0.5753 & $0.053 *$ \\
\hline $\mathrm{C} 13$ & $0.39251(14)$ & $0.06942(11)$ & $0.51413(10)$ & $0.0213(5)$ \\
\hline C14 & $0.41621(13)$ & $0.08968(11)$ & $0.45596(9)$ & $0.0204(5)$ \\
\hline $\mathrm{C} 15$ & $0.50662(15)$ & $0.10235(12)$ & $0.43992(11)$ & $0.0257(5)$ \\
\hline C16 & $0.52487(15)$ & $0.12956(15)$ & $0.38115(11)$ & $0.0377(7)$ \\
\hline H16A & 0.5842 & 0.1457 & 0.3795 & $0.057 *$ \\
\hline H16B & 0.4856 & 0.1665 & 0.3730 & $0.057^{*}$ \\
\hline $\mathrm{H} 16 \mathrm{C}$ & 0.5168 & 0.0943 & 0.3526 & $0.057^{*}$ \\
\hline C17 & $0.23544(13)$ & $0.10738(10)$ & $0.36559(9)$ & $0.0172(4)$ \\
\hline $\mathrm{C} 18$ & $0.27775(13)$ & $0.11919(11)$ & $0.31372(9)$ & $0.0191(4)$ \\
\hline H18 & 0.3385 & 0.1164 & 0.3114 & $0.023 *$ \\
\hline C19 & $0.22975(14)$ & 0.13491 (11) & 0.26627 (9) & $0.0200(5)$ \\
\hline $\mathrm{C} 20$ & $0.14114(14)$ & $0.14071(11)$ & $0.26706(10)$ & $0.0228(5)$ \\
\hline $\mathrm{H} 20$ & 0.1098 & 0.1516 & 0.2334 & $0.027 *$ \\
\hline $\mathrm{C} 21$ & $0.10001(14)$ & $0.12994(11)$ & $0.31900(10)$ & $0.0238(5)$ \\
\hline $\mathrm{H} 21$ & 0.0393 & 0.1339 & 0.3212 & $0.029^{*}$ \\
\hline $\mathrm{C} 22$ & $0.14640(13)$ & $0.11353(11)$ & $0.36762(9)$ & $0.0204(5)$ \\
\hline $\mathrm{H} 22$ & 0.1172 & 0.1064 & 0.4029 & $0.025^{*}$ \\
\hline $\mathrm{C} 23$ & $0.23529(15)$ & $-0.04049(13)$ & $0.57677(10)$ & $0.0249(5)$ \\
\hline $\mathrm{H} 23 \mathrm{~A}$ & 0.2720 & -0.0808 & 0.5752 & $0.030^{*}$ \\
\hline $\mathrm{H} 23 \mathrm{~B}$ & 0.1984 & -0.0437 & 0.6112 & $0.030^{*}$ \\
\hline $\mathrm{C} 24$ & $0.29118(15)$ & $0.02124(13)$ & $0.58082(10)$ & $0.0260(5)$ \\
\hline $\mathrm{H} 24 \mathrm{~A}$ & 0.2582 & 0.0583 & 0.5982 & $0.031 *$ \\
\hline $\mathrm{H} 24 \mathrm{~B}$ & 0.3416 & 0.0120 & 0.6055 & $0.031^{*}$ \\
\hline O1A & $0.46165(13)$ & $0.22072(15)$ & $0.78627(10)$ & $0.0664(7)$ \\
\hline $\mathrm{O} 2 \mathrm{~A}$ & $0.28475(13)$ & $0.17207(10)$ & $0.66034(9)$ & $0.0457(5)$ \\
\hline $\mathrm{O} 3 \mathrm{~A}$ & $0.04080(11)$ & $0.24229(10)$ & $0.94118(10)$ & $0.0469(5)$ \\
\hline $\mathrm{O} 4 \mathrm{~A}$ & $0.06525(13)$ & $0.25224(11)$ & $1.03186(10)$ & $0.0499(6)$ \\
\hline N1A & $0.28478(13)$ & $0.20901(10)$ & $0.80646(10)$ & $0.0296(5)$ \\
\hline $\mathrm{N} 2 \mathrm{~A}$ & $0.33228(13)$ & $0.21815(11)$ & $0.85192(10)$ & $0.0332(5)$ \\
\hline $\mathrm{H} 2 \mathrm{~A}$ & $0.3888(7)$ & $0.2245(15)$ & $0.8469(13)$ & $0.051(9)^{*}$ \\
\hline $\mathrm{N} 3 \mathrm{~A}$ & 0.08951 (14) & 0.24565 (11) & $0.98228(12)$ & $0.0370(6)$ \\
\hline $\mathrm{C} 1 \mathrm{~A}$ & $0.45036(19)$ & $0.21587(17)$ & $0.68630(13)$ & $0.0501(8)$ \\
\hline H1A1 & 0.5084 & 0.2346 & 0.6880 & $0.075^{*}$ \\
\hline H1A2 & 0.4142 & 0.2441 & 0.6619 & $0.075^{*}$ \\
\hline H1A3 & 0.4527 & 0.1707 & 0.6703 & $0.075^{*}$ \\
\hline $\mathrm{C} 2 \mathrm{~A}$ & $0.41369(17)$ & $0.21322(14)$ & $0.74488(13)$ & $0.0386(7)$ \\
\hline $\mathrm{C} 3 \mathrm{~A}$ & $0.32060(16)$ & $0.20372(12)$ & $0.75493(11)$ & $0.0295(6)$ \\
\hline $\mathrm{C} 4 \mathrm{~A}$ & $0.25922(17)$ & 0.18854 (13) & $0.70795(13)$ & $0.0341(6)$ \\
\hline $\mathrm{C} 5 \mathrm{~A}$ & $0.16462(17)$ & $0.19307(15)$ & 0.71948 (13) & $0.0423(7)$ \\
\hline H5A1 & 0.1413 & 0.2328 & 0.7006 & $0.063 *$ \\
\hline H5A2 & 0.1548 & 0.1962 & 0.7609 & $0.063 *$ \\
\hline H5A3 & 0.1360 & 0.1532 & 0.7045 & $0.063^{*}$ \\
\hline C6A & $0.29704(16)$ & 0.22805 (12) & $0.90680(11)$ & $0.0277(5)$ \\
\hline C7A & $0.20906(16)$ & $0.22976(12)$ & $0.91655(12)$ & $0.0291(6)$ \\
\hline
\end{tabular}




$\begin{array}{lllll}\text { H7A } & 0.1691 & 0.2227 & 0.8864 & 0.035^{*} \\ \text { C8A } & 0.18222(15) & 0.24215(12) & 0.97171(12) & 0.0310(6) \\ \text { C9A } & 0.23753(18) & 0.25281(14) & 1.01735(14) & 0.0367(6) \\ \text { H9A } & 0.2164 & 0.2613 & 1.0549 & 0.044^{*} \\ \text { C10A } & 0.32440(17) & 0.25058(13) & 1.00628(13) & 0.0369(6) \\ \text { H10A } & 0.3642 & 0.2577 & 1.0366 & 0.044^{*} \\ \text { C11A } & 0.35401(16) & 0.23805(13) & 0.95142(12) & 0.0330(6) \\ \text { H11A } & 0.4140 & 0.2363 & 0.9443 & 0.040^{*}\end{array}$

Atomic displacement parameters $\left(\AA^{2}\right)$

\begin{tabular}{|c|c|c|c|c|c|c|}
\hline & $U^{11}$ & $U^{22}$ & $U^{33}$ & $U^{12}$ & $U^{13}$ & $U^{23}$ \\
\hline $\mathrm{Cu} 1$ & $0.01805(14)$ & $0.02554(15)$ & $0.01264(15)$ & $-0.00435(10)$ & $-0.00054(11)$ & $0.00152(11)$ \\
\hline $\mathrm{O} 1$ & $0.0193(8)$ & $0.0433(10)$ & $0.0237(10)$ & $-0.0008(7)$ & $0.0055(7)$ & $0.0031(8)$ \\
\hline $\mathrm{O} 2$ & $0.0194(10)$ & $0.1091(19)$ & $0.0342(12)$ & $0.0037(10)$ & $-0.0026(9)$ & $0.0060(12)$ \\
\hline $\mathrm{O} 3$ & $0.0299(11)$ & 0.1047 (19) & $0.0285(12)$ & $-0.0084(11)$ & $0.0138(9)$ & $-0.0071(11)$ \\
\hline $\mathrm{O} 4$ & $0.0180(8)$ & $0.0470(11)$ & $0.0420(12)$ & $-0.0009(7)$ & $-0.0038(8)$ & $0.0096(9)$ \\
\hline O5 & $0.0451(11)$ & $0.0437(11)$ & $0.0155(9)$ & $0.0029(8)$ & $-0.0008(8)$ & $0.0026(8)$ \\
\hline O6 & $0.0291(10)$ & $0.0713(14)$ & $0.0254(11)$ & $0.0017(9)$ & $0.0110(8)$ & $0.0074(9)$ \\
\hline N1 & $0.0152(9)$ & $0.0209(9)$ & $0.0152(10)$ & $-0.0009(7)$ & $0.0019(7)$ & $0.0002(7)$ \\
\hline $\mathrm{N} 2$ & $0.0149(9)$ & $0.0263(10)$ & $0.0132(10)$ & $-0.0015(7)$ & $0.0019(7)$ & $-0.0010(7)$ \\
\hline N3 & $0.0193(10)$ & $0.0425(13)$ & $0.0261(12)$ & $0.0009(8)$ & $0.0057(9)$ & $-0.0055(9)$ \\
\hline N4 & $0.0166(9)$ & $0.0229(10)$ & $0.0197(10)$ & $-0.0026(7)$ & $0.0023(7)$ & $-0.0014(7)$ \\
\hline N5 & $0.0160(9)$ & $0.0259(10)$ & $0.0122(9)$ & $-0.0009(7)$ & $0.0000(7)$ & $-0.0001(7)$ \\
\hline N6 & $0.0328(12)$ & $0.0263(10)$ & $0.0140(11)$ & $-0.0034(8)$ & $0.0029(9)$ & $-0.0022(8)$ \\
\hline N7 & $0.0187(9)$ & $0.0287(10)$ & $0.0125(10)$ & $-0.0021(7)$ & $-0.0001(7)$ & $0.0017(7)$ \\
\hline N8 & $0.0201(9)$ & $0.0283(10)$ & $0.0139(10)$ & $-0.0030(8)$ & $-0.0017(7)$ & $0.0000(8)$ \\
\hline $\mathrm{C} 1$ & $0.0287(13)$ & $0.0370(14)$ & $0.0189(13)$ & $-0.0090(10)$ & $0.0002(10)$ & $0.0064(10)$ \\
\hline $\mathrm{C} 2$ & $0.0189(11)$ & $0.0234(11)$ & $0.0142(11)$ & $0.0018(8)$ & $0.0021(8)$ & $0.0008(8)$ \\
\hline $\mathrm{C} 3$ & $0.0165(10)$ & $0.0212(11)$ & $0.0151(11)$ & $-0.0001(8)$ & $0.0018(9)$ & $0.0005(9)$ \\
\hline $\mathrm{C} 4$ & $0.0176(11)$ & $0.0249(12)$ & $0.0184(12)$ & $-0.0002(8)$ & $0.0006(9)$ & $0.0036(9)$ \\
\hline $\mathrm{C} 5$ & $0.0186(12)$ & $0.0507(16)$ & $0.0251(14)$ & $-0.0082(11)$ & $-0.0044(10)$ & $0.0038(11)$ \\
\hline C6 & $0.0196(10)$ & $0.0191(10)$ & $0.0138(11)$ & $-0.0022(8)$ & $0.0021(9)$ & $-0.0005(8)$ \\
\hline $\mathrm{C} 7$ & $0.0177(10)$ & $0.0251(11)$ & $0.0144(11)$ & $0.0009(9)$ & $-0.0019(9)$ & $-0.0019(9)$ \\
\hline C8 & $0.0162(10)$ & $0.0283(12)$ & $0.0196(12)$ & $-0.0002(9)$ & $0.0032(9)$ & $-0.0038(9)$ \\
\hline $\mathrm{C} 9$ & $0.0269(12)$ & $0.0280(12)$ & $0.0124(11)$ & $-0.0011(9)$ & $0.0047(9)$ & $-0.0022(9)$ \\
\hline $\mathrm{C} 10$ & $0.0253(12)$ & $0.0276(12)$ & $0.0150(12)$ & $0.0004(9)$ & $-0.0023(9)$ & $-0.0012(9)$ \\
\hline C11 & $0.0170(11)$ & $0.0245(11)$ & $0.0197(12)$ & -0.0009 (8) & -0.0009 (9) & $-0.0009(9)$ \\
\hline $\mathrm{C} 12$ & $0.0307(14)$ & $0.0470(16)$ & $0.0275(15)$ & $-0.0145(12)$ & $-0.0082(11)$ & $0.0006(12)$ \\
\hline $\mathrm{C} 13$ & $0.0202(11)$ & $0.0237(12)$ & $0.0200(12)$ & $-0.0013(8)$ & $-0.0007(9)$ & 0.0009 (9) \\
\hline $\mathrm{C} 14$ & $0.0166(10)$ & $0.0246(12)$ & $0.0201(13)$ & $-0.0016(8)$ & $-0.0011(9)$ & $-0.0011(9)$ \\
\hline C15 & $0.0211(12)$ & $0.0276(13)$ & $0.0284(14)$ & $-0.0041(9)$ & $0.0002(10)$ & $-0.0010(10)$ \\
\hline $\mathrm{C} 16$ & $0.0212(12)$ & $0.0599(19)$ & $0.0320(16)$ & $-0.0085(12)$ & $0.0048(11)$ & $0.0052(13)$ \\
\hline $\mathrm{C} 17$ & $0.0181(10)$ & $0.0183(10)$ & $0.0152(11)$ & $-0.0027(8)$ & $-0.0004(9)$ & $-0.0016(8)$ \\
\hline $\mathrm{C} 18$ & $0.0179(11)$ & $0.0203(11)$ & $0.0191(12)$ & $-0.0031(8)$ & $0.0020(9)$ & $-0.0006(9)$ \\
\hline C19 & $0.0273(12)$ & $0.0196(11)$ & $0.0132(11)$ & $-0.0037(9)$ & $0.0043(9)$ & $0.0001(8)$ \\
\hline $\mathrm{C} 20$ & $0.0242(12)$ & $0.0240(11)$ & $0.0202(13)$ & $0.0011(9)$ & $-0.0042(9)$ & $0.0025(9)$ \\
\hline $\mathrm{C} 21$ & 0.0189 (11) & $0.0285(13)$ & $0.0240(13)$ & $0.0024(9)$ & $0.0014(9)$ & $0.0015(10)$ \\
\hline
\end{tabular}




\begin{tabular}{lllllll} 
& & & & & \\
C22 & $0.0200(11)$ & $0.0245(11)$ & $0.0168(12)$ & $-0.0001(8)$ & $0.0051(9)$ & $0.0020(9)$ \\
C23 & $0.0241(11)$ & $0.0348(13)$ & $0.0157(12)$ & $-0.0046(10)$ & $-0.0034(10)$ & $0.0058(10)$ \\
C24 & $0.0232(11)$ & $0.0399(14)$ & $0.0148(12)$ & $-0.0082(10)$ & $-0.0012(10)$ & $-0.0009(10)$ \\
O1A & $0.0253(11)$ & $0.125(2)$ & $0.0487(15)$ & $-0.0036(13)$ & $0.0007(10)$ & $-0.0065(14)$ \\
O2A & $0.0480(12)$ & $0.0499(13)$ & $0.0391(13)$ & $-0.0020(9)$ & $-0.0019(10)$ & $-0.0082(10)$ \\
O3A & $0.0217(10)$ & $0.0523(13)$ & $0.0666(16)$ & $0.0003(8)$ & $-0.0052(10)$ & $-0.0050(11)$ \\
O4A & $0.0394(12)$ & $0.0507(13)$ & $0.0598(16)$ & $-0.0008(9)$ & $0.0198(11)$ & $-0.0019(10)$ \\
N1A & $0.0268(11)$ & $0.0212(10)$ & $0.0408(14)$ & $0.0010(8)$ & $-0.0017(10)$ & $0.0022(9)$ \\
N2A & $0.0194(11)$ & $0.0390(13)$ & $0.0413(15)$ & $0.0025(9)$ & $-0.0025(10)$ & $0.0045(10)$ \\
N3A & $0.0273(12)$ & $0.0277(12)$ & $0.0561(17)$ & $-0.0010(9)$ & $0.0066(12)$ & $-0.0004(11)$ \\
C1A & $0.0426(17)$ & $0.058(2)$ & $0.050(2)$ & $-0.0103(14)$ & $0.0105(15)$ & $-0.0140(15)$ \\
C2A & $0.0309(15)$ & $0.0421(16)$ & $0.0430(18)$ & $0.0001(12)$ & $0.0060(13)$ & $-0.0037(13)$ \\
C3A & $0.0293(13)$ & $0.0230(12)$ & $0.0363(16)$ & $0.0030(9)$ & $0.0015(11)$ & $-0.0006(10)$ \\
C4A & $0.0338(15)$ & $0.0234(13)$ & $0.0451(18)$ & $0.0008(10)$ & $-0.0033(13)$ & $0.0007(11)$ \\
C5A & $0.0324(15)$ & $0.0404(16)$ & $0.054(2)$ & $-0.0016(11)$ & $-0.0050(13)$ & $-0.0117(14)$ \\
C6A & $0.0250(12)$ & $0.0260(13)$ & $0.0321(15)$ & $0.0032(9)$ & $0.0007(11)$ & $0.0049(10)$ \\
C7A & $0.0235(12)$ & $0.0246(12)$ & $0.0392(16)$ & $0.0001(9)$ & $-0.0047(11)$ & $0.0025(10)$ \\
C8A & $0.0193(12)$ & $0.0253(13)$ & $0.0483(18)$ & $0.0015(9)$ & $0.0018(12)$ & $0.0050(11)$ \\
C9A & $0.0367(15)$ & $0.0365(15)$ & $0.0369(16)$ & $0.0031(11)$ & $0.0011(12)$ & $0.0034(12)$ \\
C10A & $0.0309(14)$ & $0.0410(15)$ & $0.0388(17)$ & $0.0013(11)$ & $-0.0111(13)$ & $0.0055(12)$ \\
C11A & $0.0218(12)$ & $0.0347(14)$ & $0.0426(18)$ & $0.0023(10)$ & $-0.0012(11)$ & $0.0072(11)$ \\
& & & & & & \\
\hline
\end{tabular}

Geometric parameters $\left(\AA,{ }^{\circ}\right)$

\begin{tabular}{llll}
\hline $\mathrm{Cu} 1-\mathrm{N} 7$ & $1.9395(18)$ & $\mathrm{C} 15-\mathrm{C} 16$ & $1.502(3)$ \\
$\mathrm{Cu} 1-\mathrm{N} 8$ & $1.9434(18)$ & $\mathrm{C} 16-\mathrm{H} 16 \mathrm{~A}$ & 0.9800 \\
$\mathrm{Cu} 1-\mathrm{N} 2$ & $1.9527(18)$ & $\mathrm{C} 16-\mathrm{H} 16 \mathrm{~B}$ & 0.9800 \\
$\mathrm{Cu} 1-\mathrm{N} 5$ & $1.9573(18)$ & $\mathrm{C} 16-\mathrm{H} 16 \mathrm{C}$ & 0.9800 \\
$\mathrm{O} 1-\mathrm{C} 4$ & $1.221(3)$ & $\mathrm{C} 17-\mathrm{C} 22$ & $1.394(3)$ \\
$\mathrm{O} 2-\mathrm{N} 3$ & $1.207(3)$ & $\mathrm{C} 17-\mathrm{C} 18$ & $1.397(3)$ \\
$\mathrm{O} 3-\mathrm{N} 3$ & $1.220(3)$ & $\mathrm{C} 18-\mathrm{C} 19$ & $1.372(3)$ \\
$\mathrm{O} 4-\mathrm{C} 15$ & $1.225(3)$ & $\mathrm{C} 18-\mathrm{H} 18$ & 0.9500 \\
$\mathrm{O} 5-\mathrm{N} 6$ & $1.223(2)$ & $\mathrm{C} 19-\mathrm{C} 20$ & $1.386(3)$ \\
$\mathrm{O} 6-\mathrm{N} 6$ & $1.224(3)$ & $\mathrm{C} 20-\mathrm{C} 21$ & $1.387(3)$ \\
$\mathrm{N} 1-\mathrm{N} 2$ & $1.309(2)$ & $\mathrm{C} 20-\mathrm{H} 20$ & 0.9500 \\
$\mathrm{~N} 1-\mathrm{C} 3$ & $1.334(3)$ & $\mathrm{C} 21-\mathrm{C} 22$ & $1.384(3)$ \\
$\mathrm{N} 2-\mathrm{C} 6$ & $1.424(3)$ & $\mathrm{C} 21-\mathrm{H} 21$ & 0.9500 \\
$\mathrm{~N} 3-\mathrm{C} 8$ & $1.473(3)$ & $\mathrm{C} 22-\mathrm{H} 22$ & 0.9500 \\
$\mathrm{~N} 4-\mathrm{N} 5$ & $1.315(2)$ & $\mathrm{C} 23-\mathrm{C} 24$ & $1.516(3)$ \\
$\mathrm{N} 4-\mathrm{C} 14$ & $1.326(3)$ & $\mathrm{C} 23-\mathrm{H} 23 \mathrm{~A}$ & 0.9900 \\
$\mathrm{~N} 5-\mathrm{C} 17$ & $1.421(3)$ & $\mathrm{C} 23-\mathrm{H} 23 \mathrm{~B}$ & 0.9900 \\
$\mathrm{~N} 6-\mathrm{C} 19$ & $1.470(3)$ & $\mathrm{C} 24-\mathrm{H} 24 \mathrm{~A}$ & 0.9900 \\
$\mathrm{~N} 7-\mathrm{C} 2$ & $1.296(3)$ & $\mathrm{C} 24-\mathrm{H} 24 \mathrm{~B}$ & 0.9900 \\
$\mathrm{~N} 7-\mathrm{C} 23$ & $1.469(3)$ & $\mathrm{O} 1 \mathrm{~A}-\mathrm{C} 2 \mathrm{~A}$ & $1.230(3)$ \\
$\mathrm{N} 8-\mathrm{C} 13$ & $1.292(3)$ & $\mathrm{O} 2 \mathrm{~A}-\mathrm{C} 4 \mathrm{~A}$ & $1.224(3)$ \\
$\mathrm{N} 8-\mathrm{C} 24$ & $1.471(3)$ & $\mathrm{O} 3 \mathrm{~A}-\mathrm{N} 3 \mathrm{~A}$ & $1.224(3)$ \\
$\mathrm{C} 1-\mathrm{C} 2$ & $1.513(3)$ & $\mathrm{O} 4 \mathrm{~A}-\mathrm{N} 3 \mathrm{~A}$ & $1.223(3)$ \\
$\mathrm{C} 1-\mathrm{H} 1 \mathrm{~A}$ & 0.9800 & $\mathrm{~N} 1 \mathrm{~A}-\mathrm{N} 2 \mathrm{~A}$ & $1.305(3)$ \\
& & &
\end{tabular}




\begin{tabular}{|c|c|c|c|}
\hline $\mathrm{C} 1-\mathrm{H} 1 \mathrm{~B}$ & 0.9800 & $\mathrm{~N} 1 \mathrm{~A}-\mathrm{C} 3 \mathrm{~A}$ & $1.329(3)$ \\
\hline $\mathrm{C} 1-\mathrm{H} 1 \mathrm{C}$ & 0.9800 & $\mathrm{~N} 2 \mathrm{~A}-\mathrm{C} 6 \mathrm{~A}$ & $1.406(3)$ \\
\hline $\mathrm{C} 2-\mathrm{C} 3$ & $1.459(3)$ & $\mathrm{N} 2 \mathrm{~A}-\mathrm{H} 2 \mathrm{~A}$ & $0.898(10)$ \\
\hline $\mathrm{C} 3-\mathrm{C} 4$ & $1.477(3)$ & $\mathrm{N} 3 \mathrm{~A}-\mathrm{C} 8 \mathrm{~A}$ & $1.467(3)$ \\
\hline $\mathrm{C} 4-\mathrm{C} 5$ & $1.503(3)$ & $\mathrm{C} 1 \mathrm{~A}-\mathrm{C} 2 \mathrm{~A}$ & $1.481(4)$ \\
\hline $\mathrm{C} 5-\mathrm{H} 5 \mathrm{~A}$ & 0.9800 & $\mathrm{C} 1 \mathrm{~A}-\mathrm{H} 1 \mathrm{~A} 1$ & 0.9800 \\
\hline $\mathrm{C} 5-\mathrm{H} 5 \mathrm{~B}$ & 0.9800 & $\mathrm{C} 1 \mathrm{~A}-\mathrm{H} 1 \mathrm{~A} 2$ & 0.9800 \\
\hline $\mathrm{C} 5-\mathrm{H} 5 \mathrm{C}$ & 0.9800 & $\mathrm{C} 1 \mathrm{~A}-\mathrm{H} 1 \mathrm{~A} 3$ & 0.9800 \\
\hline $\mathrm{C} 6-\mathrm{C} 7$ & $1.391(3)$ & $\mathrm{C} 2 \mathrm{~A}-\mathrm{C} 3 \mathrm{~A}$ & $1.482(4)$ \\
\hline $\mathrm{C} 6-\mathrm{C} 11$ & $1.397(3)$ & $\mathrm{C} 3 \mathrm{~A}-\mathrm{C} 4 \mathrm{~A}$ & $1.485(4)$ \\
\hline $\mathrm{C} 7-\mathrm{C} 8$ & $1.379(3)$ & $\mathrm{C} 4 \mathrm{~A}-\mathrm{C} 5 \mathrm{~A}$ & $1.501(4)$ \\
\hline $\mathrm{C} 7-\mathrm{H} 7$ & 0.9500 & $\mathrm{C} 5 \mathrm{~A}-\mathrm{H} 5 \mathrm{~A} 1$ & 0.9800 \\
\hline $\mathrm{C} 8-\mathrm{C} 9$ & $1.386(3)$ & $\mathrm{C} 5 \mathrm{~A}-\mathrm{H} 5 \mathrm{~A} 2$ & 0.9800 \\
\hline $\mathrm{C} 9-\mathrm{C} 10$ & $1.381(3)$ & $\mathrm{C} 5 \mathrm{~A}-\mathrm{H} 5 \mathrm{~A} 3$ & 0.9800 \\
\hline C9-H9 & 0.9500 & $\mathrm{C} 6 \mathrm{~A}-\mathrm{C} 11 \mathrm{~A}$ & $1.382(4)$ \\
\hline $\mathrm{C} 10-\mathrm{C} 11$ & $1.385(3)$ & $\mathrm{C} 6 \mathrm{~A}-\mathrm{C} 7 \mathrm{~A}$ & $1.390(4)$ \\
\hline $\mathrm{C} 10-\mathrm{H} 10$ & 0.9500 & $\mathrm{C} 7 \mathrm{~A}-\mathrm{C} 8 \mathrm{~A}$ & $1.375(4)$ \\
\hline $\mathrm{C} 11-\mathrm{H} 11$ & 0.9500 & $\mathrm{C} 7 \mathrm{~A}-\mathrm{H} 7 \mathrm{~A}$ & 0.9500 \\
\hline $\mathrm{C} 12-\mathrm{C} 13$ & $1.501(3)$ & $\mathrm{C} 8 \mathrm{~A}-\mathrm{C} 9 \mathrm{~A}$ & $1.386(4)$ \\
\hline $\mathrm{C} 12-\mathrm{H} 12 \mathrm{~A}$ & 0.9800 & $\mathrm{C} 9 \mathrm{~A}-\mathrm{C} 10 \mathrm{~A}$ & $1.379(4)$ \\
\hline $\mathrm{C} 12-\mathrm{H} 12 \mathrm{~B}$ & 0.9800 & C9A-H9A & 0.9500 \\
\hline $\mathrm{C} 12-\mathrm{H} 12 \mathrm{C}$ & 0.9800 & $\mathrm{C} 10 \mathrm{~A}-\mathrm{C} 11 \mathrm{~A}$ & $1.382(4)$ \\
\hline $\mathrm{C} 13-\mathrm{C} 14$ & $1.463(3)$ & $\mathrm{C} 10 \mathrm{~A}-\mathrm{H} 10 \mathrm{~A}$ & 0.9500 \\
\hline $\mathrm{C} 14-\mathrm{C} 15$ & $1.479(3)$ & $\mathrm{C} 11 \mathrm{~A}-\mathrm{H} 11 \mathrm{~A}$ & 0.9500 \\
\hline $\mathrm{N} 7-\mathrm{Cu} 1-\mathrm{N} 8$ & $86.32(8)$ & $\mathrm{C} 15-\mathrm{C} 16-\mathrm{H} 16 \mathrm{~B}$ & 109.5 \\
\hline $\mathrm{N} 7-\mathrm{Cu} 1-\mathrm{N} 2$ & $88.42(8)$ & $\mathrm{H} 16 \mathrm{~A}-\mathrm{C} 16-\mathrm{H} 16 \mathrm{~B}$ & 109.5 \\
\hline $\mathrm{N} 8-\mathrm{Cu} 1-\mathrm{N} 2$ & $157.38(8)$ & $\mathrm{C} 15-\mathrm{C} 16-\mathrm{H} 16 \mathrm{C}$ & 109.5 \\
\hline $\mathrm{N} 7-\mathrm{Cu} 1-\mathrm{N} 5$ & $166.59(8)$ & $\mathrm{H} 16 \mathrm{~A}-\mathrm{C} 16-\mathrm{H} 16 \mathrm{C}$ & 109.5 \\
\hline $\mathrm{N} 8-\mathrm{Cu} 1-\mathrm{N} 5$ & $89.16(7)$ & $\mathrm{H} 16 \mathrm{~B}-\mathrm{C} 16-\mathrm{H} 16 \mathrm{C}$ & 109.5 \\
\hline $\mathrm{N} 2-\mathrm{Cu} 1-\mathrm{N} 5$ & $100.40(7)$ & $\mathrm{C} 22-\mathrm{C} 17-\mathrm{C} 18$ & $119.0(2)$ \\
\hline $\mathrm{N} 2-\mathrm{N} 1-\mathrm{C} 3$ & $122.21(18)$ & $\mathrm{C} 22-\mathrm{C} 17-\mathrm{N} 5$ & $118.85(18)$ \\
\hline $\mathrm{N} 1-\mathrm{N} 2-\mathrm{C} 6$ & $112.66(17)$ & $\mathrm{C} 18-\mathrm{C} 17-\mathrm{N} 5$ & $122.12(18)$ \\
\hline $\mathrm{N} 1-\mathrm{N} 2-\mathrm{Cu} 1$ & $123.59(14)$ & $\mathrm{C} 19-\mathrm{C} 18-\mathrm{C} 17$ & $118.63(19)$ \\
\hline $\mathrm{C} 6-\mathrm{N} 2-\mathrm{Cu} 1$ & $120.87(13)$ & $\mathrm{C} 19-\mathrm{C} 18-\mathrm{H} 18$ & 120.7 \\
\hline $\mathrm{O} 2-\mathrm{N} 3-\mathrm{O} 3$ & $123.4(2)$ & $\mathrm{C} 17-\mathrm{C} 18-\mathrm{H} 18$ & 120.7 \\
\hline $\mathrm{O} 2-\mathrm{N} 3-\mathrm{C} 8$ & $118.9(2)$ & $\mathrm{C} 18-\mathrm{C} 19-\mathrm{C} 20$ & $123.5(2)$ \\
\hline $\mathrm{O} 3-\mathrm{N} 3-\mathrm{C} 8$ & $117.7(2)$ & $\mathrm{C} 18-\mathrm{C} 19-\mathrm{N} 6$ & $117.53(19)$ \\
\hline $\mathrm{N} 5-\mathrm{N} 4-\mathrm{C} 14$ & $123.34(18)$ & $\mathrm{C} 20-\mathrm{C} 19-\mathrm{N} 6$ & $118.9(2)$ \\
\hline $\mathrm{N} 4-\mathrm{N} 5-\mathrm{C} 17$ & $111.82(17)$ & $\mathrm{C} 19-\mathrm{C} 20-\mathrm{C} 21$ & $117.3(2)$ \\
\hline $\mathrm{N} 4-\mathrm{N} 5-\mathrm{Cu} 1$ & $121.92(14)$ & $\mathrm{C} 19-\mathrm{C} 20-\mathrm{H} 20$ & 121.3 \\
\hline $\mathrm{C} 17-\mathrm{N} 5-\mathrm{Cu} 1$ & $122.01(13)$ & $\mathrm{C} 21-\mathrm{C} 20-\mathrm{H} 20$ & 121.3 \\
\hline $\mathrm{O} 5-\mathrm{N} 6-\mathrm{O} 6$ & $123.0(2)$ & $\mathrm{C} 22-\mathrm{C} 21-\mathrm{C} 20$ & $120.7(2)$ \\
\hline $\mathrm{O} 5-\mathrm{N} 6-\mathrm{C} 19$ & $118.68(19)$ & $\mathrm{C} 22-\mathrm{C} 21-\mathrm{H} 21$ & 119.6 \\
\hline $\mathrm{O} 6-\mathrm{N} 6-\mathrm{C} 19$ & $118.27(19)$ & $\mathrm{C} 20-\mathrm{C} 21-\mathrm{H} 21$ & 119.6 \\
\hline $\mathrm{C} 2-\mathrm{N} 7-\mathrm{C} 23$ & $121.64(18)$ & $\mathrm{C} 21-\mathrm{C} 22-\mathrm{C} 17$ & $120.9(2)$ \\
\hline $\mathrm{C} 2-\mathrm{N} 7-\mathrm{Cu} 1$ & $126.75(15)$ & $\mathrm{C} 21-\mathrm{C} 22-\mathrm{H} 22$ & 119.6 \\
\hline $\mathrm{C} 23-\mathrm{N} 7-\mathrm{Cu} 1$ & $111.48(13)$ & $\mathrm{C} 17-\mathrm{C} 22-\mathrm{H} 22$ & 119.6 \\
\hline
\end{tabular}




\begin{tabular}{|c|c|}
\hline $\mathrm{C} 13-\mathrm{N} 8-\mathrm{C} 24$ & $121.95(19)$ \\
\hline $\mathrm{C} 13-\mathrm{N} 8-\mathrm{Cu} 1$ & $126.78(16)$ \\
\hline $\mathrm{C} 24-\mathrm{N} 8-\mathrm{Cu} 1$ & $111.27(13)$ \\
\hline $\mathrm{C} 2-\mathrm{C} 1-\mathrm{H} 1 \mathrm{~A}$ & 109.5 \\
\hline $\mathrm{C} 2-\mathrm{C} 1-\mathrm{H} 1 \mathrm{~B}$ & 109.5 \\
\hline $\mathrm{H} 1 \mathrm{~A}-\mathrm{C} 1-\mathrm{H} 1 \mathrm{~B}$ & 109.5 \\
\hline $\mathrm{C} 2-\mathrm{C} 1-\mathrm{H} 1 \mathrm{C}$ & 109.5 \\
\hline $\mathrm{H} 1 \mathrm{~A}-\mathrm{C} 1-\mathrm{H} 1 \mathrm{C}$ & 109.5 \\
\hline $\mathrm{H} 1 \mathrm{~B}-\mathrm{C} 1-\mathrm{H} 1 \mathrm{C}$ & 109.5 \\
\hline $\mathrm{N} 7-\mathrm{C} 2-\mathrm{C} 3$ & $119.72(19)$ \\
\hline $\mathrm{N} 7-\mathrm{C} 2-\mathrm{C} 1$ & $120.57(19)$ \\
\hline $\mathrm{C} 3-\mathrm{C} 2-\mathrm{C} 1$ & $119.46(19)$ \\
\hline $\mathrm{N} 1-\mathrm{C} 3-\mathrm{C} 2$ & $125.46(19)$ \\
\hline $\mathrm{N} 1-\mathrm{C} 3-\mathrm{C} 4$ & $112.64(18)$ \\
\hline $\mathrm{C} 2-\mathrm{C} 3-\mathrm{C} 4$ & $121.77(19)$ \\
\hline $\mathrm{O} 1-\mathrm{C} 4-\mathrm{C} 3$ & $122.0(2)$ \\
\hline $\mathrm{O} 1-\mathrm{C} 4-\mathrm{C} 5$ & $119.6(2)$ \\
\hline $\mathrm{C} 3-\mathrm{C} 4-\mathrm{C} 5$ & $118.32(19)$ \\
\hline $\mathrm{C} 4-\mathrm{C} 5-\mathrm{H} 5 \mathrm{~A}$ & 109.5 \\
\hline $\mathrm{C} 4-\mathrm{C} 5-\mathrm{H} 5 \mathrm{~B}$ & 109.5 \\
\hline $\mathrm{H} 5 \mathrm{~A}-\mathrm{C} 5-\mathrm{H} 5 \mathrm{~B}$ & 109.5 \\
\hline $\mathrm{C} 4-\mathrm{C} 5-\mathrm{H} 5 \mathrm{C}$ & 109.5 \\
\hline $\mathrm{H} 5 \mathrm{~A}-\mathrm{C} 5-\mathrm{H} 5 \mathrm{C}$ & 109.5 \\
\hline $\mathrm{H} 5 \mathrm{~B}-\mathrm{C} 5-\mathrm{H} 5 \mathrm{C}$ & 109.5 \\
\hline $\mathrm{C} 7-\mathrm{C} 6-\mathrm{C} 11$ & $119.83(19)$ \\
\hline $\mathrm{C} 7-\mathrm{C} 6-\mathrm{N} 2$ & $117.31(18)$ \\
\hline $\mathrm{C} 11-\mathrm{C} 6-\mathrm{N} 2$ & $122.79(19)$ \\
\hline $\mathrm{C} 8-\mathrm{C} 7-\mathrm{C} 6$ & $118.0(2)$ \\
\hline $\mathrm{C} 8-\mathrm{C} 7-\mathrm{H} 7$ & 121.0 \\
\hline $\mathrm{C} 6-\mathrm{C} 7-\mathrm{H} 7$ & 121.0 \\
\hline $\mathrm{C} 7-\mathrm{C} 8-\mathrm{C} 9$ & $123.2(2)$ \\
\hline $\mathrm{C} 7-\mathrm{C} 8-\mathrm{N} 3$ & $118.4(2)$ \\
\hline $\mathrm{C} 9-\mathrm{C} 8-\mathrm{N} 3$ & $118.4(2)$ \\
\hline $\mathrm{C} 10-\mathrm{C} 9-\mathrm{C} 8$ & $117.9(2)$ \\
\hline $\mathrm{C} 10-\mathrm{C} 9-\mathrm{H} 9$ & 121.0 \\
\hline $\mathrm{C} 8-\mathrm{C} 9-\mathrm{H} 9$ & 121.0 \\
\hline $\mathrm{C} 9-\mathrm{C} 10-\mathrm{C} 11$ & $120.6(2)$ \\
\hline $\mathrm{C} 9-\mathrm{C} 10-\mathrm{H} 10$ & 119.7 \\
\hline $\mathrm{C} 11-\mathrm{C} 10-\mathrm{H} 10$ & 119.7 \\
\hline $\mathrm{C} 10-\mathrm{C} 11-\mathrm{C} 6$ & $120.3(2)$ \\
\hline $\mathrm{C} 10-\mathrm{C} 11-\mathrm{H} 11$ & 119.8 \\
\hline $\mathrm{C} 6-\mathrm{C} 11-\mathrm{H} 11$ & 119.8 \\
\hline $\mathrm{C} 13-\mathrm{C} 12-\mathrm{H} 12 \mathrm{~A}$ & 109.5 \\
\hline $\mathrm{C} 13-\mathrm{C} 12-\mathrm{H} 12 \mathrm{~B}$ & 109.5 \\
\hline $\mathrm{H} 12 \mathrm{~A}-\mathrm{C} 12-\mathrm{H} 12 \mathrm{~B}$ & 109.5 \\
\hline $\mathrm{C} 13-\mathrm{C} 12-\mathrm{H} 12 \mathrm{C}$ & 109.5 \\
\hline $\mathrm{H} 12 \mathrm{~A}-\mathrm{C} 12-\mathrm{H} 12 \mathrm{C}$ & 109.5 \\
\hline $\mathrm{H} 12 \mathrm{~B}-\mathrm{C} 12-\mathrm{H} 12 \mathrm{C}$ & \\
\hline
\end{tabular}

\begin{tabular}{|c|c|}
\hline $\mathrm{N} 7-\mathrm{C} 23-\mathrm{C} 24$ & $109.98(19)$ \\
\hline $\mathrm{N} 7-\mathrm{C} 23-\mathrm{H} 23 \mathrm{~A}$ & 109.7 \\
\hline $\mathrm{C} 24-\mathrm{C} 23-\mathrm{H} 23 \mathrm{~A}$ & 109.7 \\
\hline $\mathrm{N} 7-\mathrm{C} 23-\mathrm{H} 23 \mathrm{~B}$ & 109.7 \\
\hline $\mathrm{C} 24-\mathrm{C} 23-\mathrm{H} 23 \mathrm{~B}$ & 109.7 \\
\hline $\mathrm{H} 23 \mathrm{~A}-\mathrm{C} 23-\mathrm{H} 23 \mathrm{~B}$ & 108.2 \\
\hline $\mathrm{N} 8-\mathrm{C} 24-\mathrm{C} 23$ & $109.15(18)$ \\
\hline $\mathrm{N} 8-\mathrm{C} 24-\mathrm{H} 24 \mathrm{~A}$ & 109.8 \\
\hline $\mathrm{C} 23-\mathrm{C} 24-\mathrm{H} 24 \mathrm{~A}$ & 109.8 \\
\hline $\mathrm{N} 8-\mathrm{C} 24-\mathrm{H} 24 \mathrm{~B}$ & 109.8 \\
\hline $\mathrm{C} 23-\mathrm{C} 24-\mathrm{H} 24 \mathrm{~B}$ & 109.8 \\
\hline $\mathrm{H} 24 \mathrm{~A}-\mathrm{C} 24-\mathrm{H} 24 \mathrm{~B}$ & 108.3 \\
\hline $\mathrm{N} 2 \mathrm{~A}-\mathrm{N} 1 \mathrm{~A}-\mathrm{C} 3 \mathrm{~A}$ & $120.5(2)$ \\
\hline $\mathrm{N} 1 \mathrm{~A}-\mathrm{N} 2 \mathrm{~A}-\mathrm{C} 6 \mathrm{~A}$ & $122.5(2)$ \\
\hline $\mathrm{N} 1 \mathrm{~A}-\mathrm{N} 2 \mathrm{~A}-\mathrm{H} 2 \mathrm{~A}$ & $118(2)$ \\
\hline $\mathrm{C} 6 \mathrm{~A}-\mathrm{N} 2 \mathrm{~A}-\mathrm{H} 2 \mathrm{~A}$ & $119(2)$ \\
\hline $\mathrm{O} 4 \mathrm{~A}-\mathrm{N} 3 \mathrm{~A}-\mathrm{O} 3 \mathrm{~A}$ & $123.6(2)$ \\
\hline $\mathrm{O} 4 \mathrm{~A}-\mathrm{N} 3 \mathrm{~A}-\mathrm{C} 8 \mathrm{~A}$ & $117.9(2)$ \\
\hline $\mathrm{O} 3 \mathrm{~A}-\mathrm{N} 3 \mathrm{~A}-\mathrm{C} 8 \mathrm{~A}$ & $118.5(2)$ \\
\hline $\mathrm{C} 2 \mathrm{~A}-\mathrm{C} 1 \mathrm{~A}-\mathrm{H} 1 \mathrm{~A} 1$ & 109.5 \\
\hline $\mathrm{C} 2 \mathrm{~A}-\mathrm{C} 1 \mathrm{~A}-\mathrm{H} 1 \mathrm{~A} 2$ & 109.5 \\
\hline $\mathrm{H} 1 \mathrm{~A} 1-\mathrm{C} 1 \mathrm{~A}-\mathrm{H} 1 \mathrm{~A} 2$ & 109.5 \\
\hline $\mathrm{C} 2 \mathrm{~A}-\mathrm{C} 1 \mathrm{~A}-\mathrm{H} 1 \mathrm{~A} 3$ & 109.5 \\
\hline $\mathrm{H} 1 \mathrm{~A} 1-\mathrm{C} 1 \mathrm{~A}-\mathrm{H} 1 \mathrm{~A} 3$ & 109.5 \\
\hline $\mathrm{H} 1 \mathrm{~A} 2-\mathrm{C} 1 \mathrm{~A}-\mathrm{H} 1 \mathrm{~A} 3$ & 109.5 \\
\hline $\mathrm{O} 1 \mathrm{~A}-\mathrm{C} 2 \mathrm{~A}-\mathrm{C} 1 \mathrm{~A}$ & $119.0(3)$ \\
\hline $\mathrm{O} 1 \mathrm{~A}-\mathrm{C} 2 \mathrm{~A}-\mathrm{C} 3 \mathrm{~A}$ & $119.1(3)$ \\
\hline $\mathrm{C} 1 \mathrm{~A}-\mathrm{C} 2 \mathrm{~A}-\mathrm{C} 3 \mathrm{~A}$ & $121.9(3)$ \\
\hline $\mathrm{N} 1 \mathrm{~A}-\mathrm{C} 3 \mathrm{~A}-\mathrm{C} 2 \mathrm{~A}$ & $122.9(2)$ \\
\hline $\mathrm{N} 1 \mathrm{~A}-\mathrm{C} 3 \mathrm{~A}-\mathrm{C} 4 \mathrm{~A}$ & $114.4(2)$ \\
\hline $\mathrm{C} 2 \mathrm{~A}-\mathrm{C} 3 \mathrm{~A}-\mathrm{C} 4 \mathrm{~A}$ & $122.7(2)$ \\
\hline $\mathrm{O} 2 \mathrm{~A}-\mathrm{C} 4 \mathrm{~A}-\mathrm{C} 3 \mathrm{~A}$ & $121.0(2)$ \\
\hline $\mathrm{O} 2 \mathrm{~A}-\mathrm{C} 4 \mathrm{~A}-\mathrm{C} 5 \mathrm{~A}$ & $119.8(2)$ \\
\hline $\mathrm{C} 3 \mathrm{~A}-\mathrm{C} 4 \mathrm{~A}-\mathrm{C} 5 \mathrm{~A}$ & $119.2(2)$ \\
\hline $\mathrm{C} 4 \mathrm{~A}-\mathrm{C} 5 \mathrm{~A}-\mathrm{H} 5 \mathrm{~A} 1$ & 109.5 \\
\hline $\mathrm{C} 4 \mathrm{~A}-\mathrm{C} 5 \mathrm{~A}-\mathrm{H} 5 \mathrm{~A} 2$ & 109.5 \\
\hline $\mathrm{H} 5 \mathrm{~A} 1-\mathrm{C} 5 \mathrm{~A}-\mathrm{H} 5 \mathrm{~A} 2$ & 109.5 \\
\hline $\mathrm{C} 4 \mathrm{~A}-\mathrm{C} 5 \mathrm{~A}-\mathrm{H} 5 \mathrm{~A} 3$ & 109.5 \\
\hline $\mathrm{H} 5 \mathrm{~A} 1-\mathrm{C} 5 \mathrm{~A}-\mathrm{H} 5 \mathrm{~A} 3$ & 109.5 \\
\hline $\mathrm{H} 5 \mathrm{~A} 2-\mathrm{C} 5 \mathrm{~A}-\mathrm{H} 5 \mathrm{~A} 3$ & 109.5 \\
\hline $\mathrm{C} 11 \mathrm{~A}-\mathrm{C} 6 \mathrm{~A}-\mathrm{C} 7 \mathrm{~A}$ & $120.5(2)$ \\
\hline $\mathrm{C} 11 \mathrm{~A}-\mathrm{C} 6 \mathrm{~A}-\mathrm{N} 2 \mathrm{~A}$ & $117.0(2)$ \\
\hline $\mathrm{C} 7 \mathrm{~A}-\mathrm{C} 6 \mathrm{~A}-\mathrm{N} 2 \mathrm{~A}$ & $122.5(2)$ \\
\hline $\mathrm{C} 8 \mathrm{~A}-\mathrm{C} 7 \mathrm{~A}-\mathrm{C} 6 \mathrm{~A}$ & $117.2(2)$ \\
\hline $\mathrm{C} 8 \mathrm{~A}-\mathrm{C} 7 \mathrm{~A}-\mathrm{H} 7 \mathrm{~A}$ & 121.4 \\
\hline $\mathrm{C} 6 \mathrm{~A}-\mathrm{C} 7 \mathrm{~A}-\mathrm{H} 7 \mathrm{~A}$ & 121.4 \\
\hline $\mathrm{C} 7 \mathrm{~A}-\mathrm{C} 8 \mathrm{~A}-\mathrm{C} 9 \mathrm{~A}$ & $123.8(2)$ \\
\hline $\mathrm{C} 7 \mathrm{~A}-\mathrm{C} 8 \mathrm{~A}-\mathrm{N} 3 \mathrm{~A}$ & $117.7(2)$ \\
\hline
\end{tabular}




\begin{tabular}{|c|c|c|c|}
\hline $\mathrm{N} 8-\mathrm{C} 13-\mathrm{C} 14$ & $119.7(2)$ & $\mathrm{C} 9 \mathrm{~A}-\mathrm{C} 8 \mathrm{~A}-\mathrm{N} 3 \mathrm{~A}$ & $118.4(2)$ \\
\hline $\mathrm{N} 8-\mathrm{C} 13-\mathrm{C} 12$ & $120.6(2)$ & $\mathrm{C} 10 \mathrm{~A}-\mathrm{C} 9 \mathrm{~A}-\mathrm{C} 8 \mathrm{~A}$ & $117.5(3)$ \\
\hline $\mathrm{C} 14-\mathrm{C} 13-\mathrm{C} 12$ & $119.6(2)$ & $\mathrm{C} 10 \mathrm{~A}-\mathrm{C} 9 \mathrm{~A}-\mathrm{H} 9 \mathrm{~A}$ & 121.2 \\
\hline $\mathrm{N} 4-\mathrm{C} 14-\mathrm{C} 13$ & $125.75(19)$ & $\mathrm{C} 8 \mathrm{~A}-\mathrm{C} 9 \mathrm{~A}-\mathrm{H} 9 \mathrm{~A}$ & 121.2 \\
\hline $\mathrm{N} 4-\mathrm{C} 14-\mathrm{C} 15$ & $112.74(19)$ & $\mathrm{C} 9 \mathrm{~A}-\mathrm{C} 10 \mathrm{~A}-\mathrm{C} 11 \mathrm{~A}$ & $120.4(3)$ \\
\hline $\mathrm{C} 13-\mathrm{C} 14-\mathrm{C} 15$ & $121.5(2)$ & $\mathrm{C} 9 \mathrm{~A}-\mathrm{C} 10 \mathrm{~A}-\mathrm{H} 10 \mathrm{~A}$ & 119.8 \\
\hline $\mathrm{O} 4-\mathrm{C} 15-\mathrm{C} 14$ & $122.0(2)$ & $\mathrm{C} 11 \mathrm{~A}-\mathrm{C} 10 \mathrm{~A}-\mathrm{H} 10 \mathrm{~A}$ & 119.8 \\
\hline $\mathrm{O} 4-\mathrm{C} 15-\mathrm{C} 16$ & $119.7(2)$ & $\mathrm{C} 6 \mathrm{~A}-\mathrm{C} 11 \mathrm{~A}-\mathrm{C} 10 \mathrm{~A}$ & $120.5(2)$ \\
\hline $\mathrm{C} 14-\mathrm{C} 15-\mathrm{C} 16$ & $118.2(2)$ & $\mathrm{C} 6 \mathrm{~A}-\mathrm{C} 11 \mathrm{~A}-\mathrm{H} 11 \mathrm{~A}$ & 119.7 \\
\hline $\mathrm{C} 15-\mathrm{C} 16-\mathrm{H} 16 \mathrm{~A}$ & 109.5 & $\mathrm{C} 10 \mathrm{~A}-\mathrm{C} 11 \mathrm{~A}-\mathrm{H} 11 \mathrm{~A}$ & 119.7 \\
\hline $\mathrm{C} 3-\mathrm{N} 1-\mathrm{N} 2-\mathrm{C} 6$ & $174.16(18)$ & $\mathrm{N} 4-\mathrm{N} 5-\mathrm{C} 17-\mathrm{C} 22$ & $-162.16(19)$ \\
\hline $\mathrm{C} 3-\mathrm{N} 1-\mathrm{N} 2-\mathrm{Cu} 1$ & $-25.0(3)$ & $\mathrm{Cu} 1-\mathrm{N} 5-\mathrm{C} 17-\mathrm{C} 22$ & $40.6(3)$ \\
\hline $\mathrm{C} 14-\mathrm{N} 4-\mathrm{N} 5-\mathrm{C} 17$ & 175.52 (19) & $\mathrm{N} 4-\mathrm{N} 5-\mathrm{C} 17-\mathrm{C} 18$ & $20.8(3)$ \\
\hline $\mathrm{C} 14-\mathrm{N} 4-\mathrm{N} 5-\mathrm{Cu} 1$ & $-27.2(3)$ & $\mathrm{Cu} 1-\mathrm{N} 5-\mathrm{C} 17-\mathrm{C} 18$ & $-136.36(17)$ \\
\hline $\mathrm{C} 23-\mathrm{N} 7-\mathrm{C} 2-\mathrm{C} 3$ & $-171.5(2)$ & $\mathrm{C} 22-\mathrm{C} 17-\mathrm{C} 18-\mathrm{C} 19$ & $-1.5(3)$ \\
\hline $\mathrm{Cu} 1-\mathrm{N} 7-\mathrm{C} 2-\mathrm{C} 3$ & $4.0(3)$ & $\mathrm{N} 5-\mathrm{C} 17-\mathrm{C} 18-\mathrm{C} 19$ & 175.48 (19) \\
\hline $\mathrm{C} 23-\mathrm{N} 7-\mathrm{C} 2-\mathrm{C} 1$ & $2.7(3)$ & $\mathrm{C} 17-\mathrm{C} 18-\mathrm{C} 19-\mathrm{C} 20$ & $1.0(3)$ \\
\hline $\mathrm{Cu} 1-\mathrm{N} 7-\mathrm{C} 2-\mathrm{C} 1$ & $178.17(17)$ & $\mathrm{C} 17-\mathrm{C} 18-\mathrm{C} 19-\mathrm{N} 6$ & $-176.94(18)$ \\
\hline $\mathrm{N} 2-\mathrm{N} 1-\mathrm{C} 3-\mathrm{C} 2$ & $-12.0(3)$ & $\mathrm{O} 5-\mathrm{N} 6-\mathrm{C} 19-\mathrm{C} 18$ & $-176.2(2)$ \\
\hline $\mathrm{N} 2-\mathrm{N} 1-\mathrm{C} 3-\mathrm{C} 4$ & 172.09 (19) & $\mathrm{O} 6-\mathrm{N} 6-\mathrm{C} 19-\mathrm{C} 18$ & $3.9(3)$ \\
\hline $\mathrm{N} 7-\mathrm{C} 2-\mathrm{C} 3-\mathrm{N} 1$ & $23.5(3)$ & $\mathrm{O} 5-\mathrm{N} 6-\mathrm{C} 19-\mathrm{C} 20$ & $5.9(3)$ \\
\hline $\mathrm{C} 1-\mathrm{C} 2-\mathrm{C} 3-\mathrm{N} 1$ & $-150.7(2)$ & $\mathrm{O} 6-\mathrm{N} 6-\mathrm{C} 19-\mathrm{C} 20$ & $-174.1(2)$ \\
\hline $\mathrm{N} 7-\mathrm{C} 2-\mathrm{C} 3-\mathrm{C} 4$ & $-161.0(2)$ & $\mathrm{C} 18-\mathrm{C} 19-\mathrm{C} 20-\mathrm{C} 21$ & $0.1(3)$ \\
\hline $\mathrm{C} 1-\mathrm{C} 2-\mathrm{C} 3-\mathrm{C} 4$ & $24.8(3)$ & $\mathrm{N} 6-\mathrm{C} 19-\mathrm{C} 20-\mathrm{C} 21$ & $177.9(2)$ \\
\hline $\mathrm{N} 1-\mathrm{C} 3-\mathrm{C} 4-\mathrm{O} 1$ & $-168.9(2)$ & $\mathrm{C} 19-\mathrm{C} 20-\mathrm{C} 21-\mathrm{C} 22$ & $-0.5(3)$ \\
\hline $\mathrm{C} 2-\mathrm{C} 3-\mathrm{C} 4-\mathrm{O} 1$ & $15.1(3)$ & $\mathrm{C} 20-\mathrm{C} 21-\mathrm{C} 22-\mathrm{C} 17$ & $0.0(3)$ \\
\hline $\mathrm{N} 1-\mathrm{C} 3-\mathrm{C} 4-\mathrm{C} 5$ & $7.6(3)$ & $\mathrm{C} 18-\mathrm{C} 17-\mathrm{C} 22-\mathrm{C} 21$ & $1.1(3)$ \\
\hline $\mathrm{C} 2-\mathrm{C} 3-\mathrm{C} 4-\mathrm{C} 5$ & $-168.4(2)$ & $\mathrm{N} 5-\mathrm{C} 17-\mathrm{C} 22-\mathrm{C} 21$ & $-176.0(2)$ \\
\hline $\mathrm{N} 1-\mathrm{N} 2-\mathrm{C} 6-\mathrm{C} 7$ & $-156.94(19)$ & $\mathrm{C} 2-\mathrm{N} 7-\mathrm{C} 23-\mathrm{C} 24$ & $-156.1(2)$ \\
\hline $\mathrm{Cu} 1-\mathrm{N} 2-\mathrm{C} 6-\mathrm{C} 7$ & $41.6(2)$ & $\mathrm{Cu} 1-\mathrm{N} 7-\mathrm{C} 23-\mathrm{C} 24$ & $27.8(2)$ \\
\hline $\mathrm{N} 1-\mathrm{N} 2-\mathrm{C} 6-\mathrm{C} 11$ & $26.1(3)$ & $\mathrm{C} 13-\mathrm{N} 8-\mathrm{C} 24-\mathrm{C} 23$ & $-149.4(2)$ \\
\hline $\mathrm{Cu} 1-\mathrm{N} 2-\mathrm{C} 6-\mathrm{C} 11$ & $-135.30(18)$ & $\mathrm{Cu} 1-\mathrm{N} 8-\mathrm{C} 24-\mathrm{C} 23$ & $30.5(2)$ \\
\hline $\mathrm{C} 11-\mathrm{C} 6-\mathrm{C} 7-\mathrm{C} 8$ & $3.0(3)$ & $\mathrm{N} 7-\mathrm{C} 23-\mathrm{C} 24-\mathrm{N} 8$ & $-37.7(3)$ \\
\hline $\mathrm{N} 2-\mathrm{C} 6-\mathrm{C} 7-\mathrm{C} 8$ & $-173.99(19)$ & $\mathrm{C} 3 \mathrm{~A}-\mathrm{N} 1 \mathrm{~A}-\mathrm{N} 2 \mathrm{~A}-\mathrm{C} 6 \mathrm{~A}$ & $176.1(2)$ \\
\hline $\mathrm{C} 6-\mathrm{C} 7-\mathrm{C} 8-\mathrm{C} 9$ & $-1.2(3)$ & $\mathrm{N} 2 \mathrm{~A}-\mathrm{N} 1 \mathrm{~A}-\mathrm{C} 3 \mathrm{~A}-\mathrm{C} 2 \mathrm{~A}$ & $-5.2(4)$ \\
\hline $\mathrm{C} 6-\mathrm{C} 7-\mathrm{C} 8-\mathrm{N} 3$ & $176.08(19)$ & $\mathrm{N} 2 \mathrm{~A}-\mathrm{N} 1 \mathrm{~A}-\mathrm{C} 3 \mathrm{~A}-\mathrm{C} 4 \mathrm{~A}$ & $175.8(2)$ \\
\hline $\mathrm{O} 2-\mathrm{N} 3-\mathrm{C} 8-\mathrm{C} 7$ & $3.4(3)$ & $\mathrm{O} 1 \mathrm{~A}-\mathrm{C} 2 \mathrm{~A}-\mathrm{C} 3 \mathrm{~A}-\mathrm{N} 1 \mathrm{~A}$ & $6.4(4)$ \\
\hline $\mathrm{O} 3-\mathrm{N} 3-\mathrm{C} 8-\mathrm{C} 7$ & $-176.3(2)$ & $\mathrm{C} 1 \mathrm{~A}-\mathrm{C} 2 \mathrm{~A}-\mathrm{C} 3 \mathrm{~A}-\mathrm{N} 1 \mathrm{~A}$ & $-171.8(2)$ \\
\hline $\mathrm{O} 2-\mathrm{N} 3-\mathrm{C} 8-\mathrm{C} 9$ & $-179.2(2)$ & $\mathrm{O} 1 \mathrm{~A}-\mathrm{C} 2 \mathrm{~A}-\mathrm{C} 3 \mathrm{~A}-\mathrm{C} 4 \mathrm{~A}$ & $-174.6(3)$ \\
\hline $\mathrm{O} 3-\mathrm{N} 3-\mathrm{C} 8-\mathrm{C} 9$ & $1.1(3)$ & $\mathrm{C} 1 \mathrm{~A}-\mathrm{C} 2 \mathrm{~A}-\mathrm{C} 3 \mathrm{~A}-\mathrm{C} 4 \mathrm{~A}$ & $7.2(4)$ \\
\hline $\mathrm{C} 7-\mathrm{C} 8-\mathrm{C} 9-\mathrm{C} 10$ & $-1.4(3)$ & $\mathrm{N} 1 \mathrm{~A}-\mathrm{C} 3 \mathrm{~A}-\mathrm{C} 4 \mathrm{~A}-\mathrm{O} 2 \mathrm{~A}$ & $-168.4(2)$ \\
\hline $\mathrm{N} 3-\mathrm{C} 8-\mathrm{C} 9-\mathrm{C} 10$ & $-178.7(2)$ & $\mathrm{C} 2 \mathrm{~A}-\mathrm{C} 3 \mathrm{~A}-\mathrm{C} 4 \mathrm{~A}-\mathrm{O} 2 \mathrm{~A}$ & $12.5(4)$ \\
\hline $\mathrm{C} 8-\mathrm{C} 9-\mathrm{C} 10-\mathrm{C} 11$ & $2.3(3)$ & $\mathrm{N} 1 \mathrm{~A}-\mathrm{C} 3 \mathrm{~A}-\mathrm{C} 4 \mathrm{~A}-\mathrm{C} 5 \mathrm{~A}$ & $11.1(3)$ \\
\hline $\mathrm{C} 9-\mathrm{C} 10-\mathrm{C} 11-\mathrm{C} 6$ & $-0.5(3)$ & $\mathrm{C} 2 \mathrm{~A}-\mathrm{C} 3 \mathrm{~A}-\mathrm{C} 4 \mathrm{~A}-\mathrm{C} 5 \mathrm{~A}$ & $-167.9(2)$ \\
\hline $\mathrm{C} 7-\mathrm{C} 6-\mathrm{C} 11-\mathrm{C} 10$ & $-2.2(3)$ & $\mathrm{N} 1 \mathrm{~A}-\mathrm{N} 2 \mathrm{~A}-\mathrm{C} 6 \mathrm{~A}-\mathrm{C} 11 \mathrm{~A}$ & $-179.2(2)$ \\
\hline $\mathrm{N} 2-\mathrm{C} 6-\mathrm{C} 11-\mathrm{C} 10$ & $174.6(2)$ & $\mathrm{N} 1 \mathrm{~A}-\mathrm{N} 2 \mathrm{~A}-\mathrm{C} 6 \mathrm{~A}-\mathrm{C} 7 \mathrm{~A}$ & $-0.9(4)$ \\
\hline $\mathrm{C} 24-\mathrm{N} 8-\mathrm{C} 13-\mathrm{C} 14$ & $-177.5(2)$ & $\mathrm{C} 11 \mathrm{~A}-\mathrm{C} 6 \mathrm{~A}-\mathrm{C} 7 \mathrm{~A}-\mathrm{C} 8 \mathrm{~A}$ & $0.5(3)$ \\
\hline
\end{tabular}




$\begin{array}{llll}\mathrm{Cu} 1-\mathrm{N} 8-\mathrm{C} 13-\mathrm{C} 14 & 2.6(3) & \mathrm{N} 2 \mathrm{~A}-\mathrm{C} 6 \mathrm{~A}-\mathrm{C} 7 \mathrm{~A}-\mathrm{C} 8 \mathrm{~A} & -177.7(2) \\ \mathrm{C} 24-\mathrm{N} 8-\mathrm{C} 13-\mathrm{C} 12 & -2.3(3) & \mathrm{C} 6 \mathrm{~A}-\mathrm{C} 7 \mathrm{~A}-\mathrm{C} 8 \mathrm{~A}-\mathrm{C} 9 \mathrm{~A} & 0.0(4) \\ \mathrm{Cu} 1-\mathrm{N} 8-\mathrm{C} 13-\mathrm{C} 12 & 177.78(18) & \mathrm{C} 6 \mathrm{~A}-\mathrm{C} 7 \mathrm{~A}-\mathrm{C} 8 \mathrm{~A}-\mathrm{N} 3 \mathrm{~A} & 178.6(2) \\ \mathrm{N} 5-\mathrm{N} 4-\mathrm{C} 14-\mathrm{C} 13 & -8.6(3) & \mathrm{O} 4 \mathrm{~A}-\mathrm{N} 3 \mathrm{~A}-\mathrm{C} 8 \mathrm{~A}-\mathrm{C} 7 \mathrm{~A} & 175.2(2) \\ \mathrm{N} 5-\mathrm{N} 4-\mathrm{C} 14-\mathrm{C} 15 & 173.19(19) & \mathrm{O} 3 \mathrm{~A}-\mathrm{N} 3 \mathrm{~A}-\mathrm{C} 8 \mathrm{~A}-\mathrm{C} 7 \mathrm{~A} & -5.2(3) \\ \mathrm{N} 8-\mathrm{C} 13-\mathrm{C} 14-\mathrm{N} 4 & 22.3(4) & \mathrm{O} 4 \mathrm{~A}-\mathrm{N} 3 \mathrm{~A}-\mathrm{C} 8 \mathrm{~A}-\mathrm{C} 9 \mathrm{~A} & -6.1(3) \\ \mathrm{C} 12-\mathrm{C} 13-\mathrm{C} 14-\mathrm{N} 4 & -153.0(2) & \mathrm{O} 3 \mathrm{~A}-\mathrm{N} 3 \mathrm{~A}-\mathrm{C} 8 \mathrm{~A}-\mathrm{C} 9 \mathrm{~A} & 173.5(2) \\ \mathrm{N} 8-\mathrm{C} 13-\mathrm{C} 14-\mathrm{C} 15 & -159.6(2) & \mathrm{C} 7 \mathrm{~A}-\mathrm{C} 8 \mathrm{~A}-\mathrm{C} 9 \mathrm{~A}-\mathrm{C} 10 \mathrm{~A} & -0.2(4) \\ \mathrm{C} 12-\mathrm{C} 13-\mathrm{C} 14-\mathrm{C} 15 & 25.1(3) & \mathrm{N} 3 \mathrm{~A}-\mathrm{C} 8 \mathrm{~A}-\mathrm{C} 9 \mathrm{~A}-\mathrm{C} 10 \mathrm{~A} & -178.8(2) \\ \mathrm{N} 4-\mathrm{C} 14-\mathrm{C} 15-\mathrm{O} 4 & -172.4(2) & \mathrm{C} 8 \mathrm{~A}-\mathrm{C} 9 \mathrm{~A}-\mathrm{C} 10 \mathrm{~A}-\mathrm{C} 11 \mathrm{~A} & -0.1(4) \\ \mathrm{C} 13-\mathrm{C} 14-\mathrm{C} 15-\mathrm{O} 4 & 9.2(4) & \mathrm{C} 7 \mathrm{~A}-\mathrm{C} 6 \mathrm{~A}-\mathrm{C} 11 \mathrm{~A}-\mathrm{C} 10 \mathrm{~A} & -0.7(4) \\ \mathrm{N} 4-\mathrm{C} 14-\mathrm{C} 15-\mathrm{C} 16 & 4.4(3) & \mathrm{N} 2 \mathrm{~A}-\mathrm{C} 6 \mathrm{~A}-\mathrm{C} 11 \mathrm{~A}-\mathrm{C} 10 \mathrm{~A} & 177.6(2) \\ \mathrm{C} 13-\mathrm{C} 14-\mathrm{C} 15-\mathrm{C} 16 & -174.0(2) & \mathrm{C} 9 \mathrm{~A}-\mathrm{C} 10 \mathrm{~A}-\mathrm{C} 11 \mathrm{~A}-\mathrm{C} 6 \mathrm{~A} & 0.5(4)\end{array}$

Hydrogen-bond geometry $\left(\AA,{ }^{\circ}\right)$

\begin{tabular}{lllll}
\hline$D-\mathrm{H} \cdots A$ & $D-\mathrm{H}$ & $\mathrm{H} \cdots A$ & $D \cdots A$ & $D-\mathrm{H}^{\cdots} A$ \\
\hline $\mathrm{C} 11 A-\mathrm{H} 11 A \cdots \mathrm{O} 4 A^{\mathrm{i}}$ & 0.95 & 2.43 & $3.320(3)$ & 156 \\
$\mathrm{~N} 2 A-\mathrm{H} 2 A \cdots \mathrm{O} 1 A$ & $0.90(1)$ & $1.81(2)$ & $2.531(3)$ & $135(3)$ \\
$\mathrm{C} 22-\mathrm{H} 22 \cdots \mathrm{O} 1^{\mathrm{ii}}$ & 0.95 & 2.27 & $3.207(3)$ & 169 \\
$\mathrm{C} 16-\mathrm{H} 16 A \cdots \mathrm{O} 5^{\mathrm{iii}}$ & 0.98 & 2.63 & $3.532(3)$ & 154 \\
$\mathrm{C} 12-\mathrm{H} 12 B \cdots \mathrm{O} 3 A^{\mathrm{iv}}$ & 0.98 & 2.53 & $3.413(3)$ & 151 \\
$\mathrm{C} 11-\mathrm{H} 11 \cdots 3^{\mathrm{v}}$ & 0.95 & 2.50 & $3.350(3)$ & 150 \\
$\mathrm{C} 5-\mathrm{H} 5 C^{\cdots} \cdots 3^{\mathrm{v}}$ & 0.98 & 2.65 & $3.426(3)$ & 137 \\
$\mathrm{C} 1-\mathrm{H} 1 B \cdots \mathrm{O} 3 A^{\mathrm{vi}}$ & 0.98 & 2.58 & $3.490(3)$ & 154 \\
$\mathrm{C} 1-\mathrm{H} 1 B \cdots \mathrm{O} 1$ & 0.98 & 2.34 & $2.842(3)$ & 111
\end{tabular}

Symmetry codes: (i) $x+1 / 2,-y+1 / 2,-z+2$; (ii) $-x,-y,-z+1$; (iii) $x+1 / 2, y,-z+1 / 2$; (iv) $x+1 / 2, y,-z+3 / 2$; (v) $x-1 / 2, y,-z+1 / 2$; (vi) $-x, y-1 / 2,-z+3 / 2$. 